\title{
Duality of matrix-weighted Besov spaces
}

\author{
by \\ Svetlana Roudenko (Durham, NC)
}

\begin{abstract}
We determine the duals of the homogeneous matrix-weighted Besov spaces $\dot{B}_{p}^{\alpha q}(W)$ and $\dot{b}_{p}^{\alpha q}(W)$ which were previously defined in [5]. If $W$ is a matrix $A_{p}$ weight, then the dual of $\dot{B}_{p}^{\alpha q}(W)$ can be identified with $\dot{B}_{p^{\prime}}^{-\alpha q^{\prime}}\left(W^{-p^{\prime} / p}\right)$ and, similarly, $\left[\dot{b}_{p}^{\alpha q}(W)\right]^{*} \approx$ $\dot{b}_{p^{\prime}}^{-\alpha q^{\prime}}\left(W^{-p^{\prime} / p}\right)$. Moreover, for certain $W$ which may not be in the $A_{p}$ class, the duals of $\dot{B}_{p}^{\alpha q}(W)$ and $\dot{b}_{p}^{\alpha q}(W)$ are determined and expressed in terms of the Besov spaces $\dot{B}_{p^{\prime}}^{-\alpha q^{\prime}}\left(\left\{A_{Q}^{-1}\right\}\right)$ and $\dot{b}_{p^{\prime}}^{-\alpha q^{\prime}}\left(\left\{A_{Q}^{-1}\right\}\right)$, which we define in terms of reducing operators $\left\{A_{Q}\right\}_{Q}$ associated with $W$. We also develop the basic theory of these reducing operator Besov spaces. Similar results are shown for inhomogeneous spaces.
\end{abstract}

1. Introduction. The aim of this paper is to determine the duals of the Besov function spaces $\dot{B}_{p}^{\alpha q}(W)$ and the corresponding sequence spaces $\dot{b}_{p}^{\alpha q}(W)$ for $\alpha \in \mathbb{R}, 0<q<\infty$ and $1<p<\infty$. Here, $W$ is a matrix weight, namely, an a.e. invertible map from $\mathbb{R}^{n}$ to the cone $\mathcal{M}$ of non-negative definite operators on a Hilbert space $\mathcal{H}$ of dimension $m$ (e.g. $\mathcal{H}=\mathbb{C}^{m}$ or $\left.\mathbb{R}^{m}\right)$, i.e., for a.e. $t \in \mathbb{R}^{n},(W(t) x, x)_{\mathcal{H}} \geq 0$ for all $x \in \mathcal{H}$.

To understand what properties of $W$ are needed to identify dual spaces, we will heavily use the technique of reducing operators (for definitions refer to Section 2 or [5], [10]). Namely, instead of dealing with matrix weights, we consider a sequence of matrices enumerated by dyadic cubes and establish properties of Besov spaces with such sequences of matrix weights. Then, given a matrix $W$, its reducing operators constitute such a sequence.

Denote by $\mathcal{D}$ the collection of dyadic cubes in $\mathbb{R}^{n}$ and for each $Q \in \mathcal{D}$ let $A_{Q}$ be a positive-definite (thus, self-adjoint) operator on $\mathcal{H}$. Also denote by $\mathcal{R} \mathcal{S}_{\mathcal{D}}$ (reducing sequences) the collection of all sequences $\left\{A_{Q}\right\}_{Q \in \mathcal{D}}$ of positive-definite operators on $\mathcal{H}$. An admissible kernel $\varphi \in \mathcal{A}$ is a function $\varphi \in \mathcal{S}\left(\mathbb{R}^{n}\right)$ such that $\operatorname{supp} \widehat{\varphi} \subseteq\left\{\xi \in \mathbb{R}^{n}: 1 / 2 \leq|\xi| \leq 2\right\}$ and $|\widehat{\varphi}(\xi)| \geq c>0$ if $3 / 5 \leq|\xi| \leq 5 / 3$. Set $\varphi_{\nu}(x)=2^{\nu n} \varphi\left(2^{\nu} x\right)$ for $\nu \in \mathbb{Z}$.

2000 Mathematics Subject Classification: Primary 42B25, 42B35, 46A20, 46B10; Secondary 47B37, 47B38.

Key words and phrases: duality, Besov spaces, matrix weights, reducing operators, $A_{p}$ condition, doubling measure, $\varphi$-transform. 
In [5] we introduced the following:

Definition 1.1 (Matrix-weighted Besov space $\dot{B}_{p}^{\alpha q}(W)$ ). For $\alpha \in \mathbb{R}$, $1 \leq p<\infty, 0<q \leq \infty, \varphi \in \mathcal{A}$ and $W$ a matrix weight, the Besov space $\dot{B}_{p}^{\alpha q}(W)$ is the collection of all vector-valued distributions $\vec{f}=\left(f_{1}, \ldots, f_{m}\right)^{\mathrm{T}}$ with $f_{i} \in \mathcal{S}^{\prime} / \mathcal{P}\left(\mathbb{R}^{n}\right)$ (the space of tempered distributions modulo polynomials), $1 \leq i \leq m$, such that

$$
\begin{aligned}
\|\vec{f}\|_{\dot{B}_{p}^{\alpha q}(W)} & =\left\|\left\{2^{\nu \alpha}\left\|\varphi_{\nu} * \vec{f}\right\|_{L^{p}(W)}\right\}_{\nu}\right\|_{l^{q}} \\
& =\left\|\left\{\left\|W^{1 / p}(t) \cdot\left(\varphi_{\nu} * \vec{f}\right)(t)\right\|_{L^{p}(d t)}\right\}_{\nu}\right\|_{l_{q}^{\alpha}}<\infty,
\end{aligned}
$$

where $\varphi_{\nu} * \vec{f}=\left(\varphi_{\nu} * f_{1}, \ldots, \varphi_{\nu} * f_{m}\right)^{\mathrm{T}}$.

Suppose $W$ satisfies any of the three conditions:

(A1) $W \in A_{p}$ with $1<p<\infty$,

(A2) $\quad W$ is a doubling matrix of order $p$ with $p>\beta$, where $\beta$ is the doubling exponent of $W$,

(A3) $W$ is a diagonal doubling matrix of order $p$ with $1 \leq p<\infty$.

(For definitions refer to Section 2.) Then $\dot{B}_{p}^{\alpha q}(W)$ is independent of the choice of $\varphi \in \mathcal{A}$ ([5, Theorem 1.8]). If a matrix weight $W$ satisfies none of (A1)-(A3), then there may be a dependence on $\varphi$ (i.e., $\dot{B}_{p}^{\alpha q}(W, \varphi)$ ), nevertheless, all results will hold up to a choice of an admissible kernel $\varphi$.

Here, as a main tool and a useful object by itself, we define the space $\dot{B}_{p}^{\alpha q}\left(\left\{A_{Q}\right\}\right)$ with a sequence of discrete weights $\left\{A_{Q}\right\}_{Q}$ :

Definition 1.2 (Averaging matrix-weighted Besov space $\dot{B}_{p}^{\alpha q}\left(\left\{A_{Q}\right\}\right)$ ). For $\alpha \in \mathbb{R}, 1 \leq p \leq \infty, 0<q \leq \infty,\left\{A_{Q}\right\}_{Q} \in \mathcal{R} \mathcal{S}_{\mathcal{D}}$ and $\varphi \in \mathcal{A}$, the Besov space $\dot{B}_{p}^{\alpha q}\left(\left\{A_{Q}\right\}\right)$ is the collection of all vector-valued distributions $\vec{f}=\left(f_{1}, \ldots, f_{m}\right)^{\mathrm{T}}$ with $f_{i} \in \mathcal{S}^{\prime} / \mathcal{P}\left(\mathbb{R}^{n}\right), 1 \leq i \leq m$, such that

$$
\|\vec{f}\|_{\dot{B}_{p}^{\alpha q}\left(\left\{A_{Q}\right\}\right)}=\left\|\left\{2^{\nu \alpha}\left\|\sum_{l(Q)=2^{-\nu}}\right\| A_{Q} \cdot\left(\varphi_{\nu} * \vec{f}\right)\left\|_{\mathcal{H}^{\prime}} \chi_{Q}\right\|_{L^{p}}\right\}_{\nu}\right\|_{l^{q}}<\infty
$$

where $l(Q)$ is the side length of $Q$.

This space is well defined (i.e., independent of $\varphi \in \mathcal{A}$ ), see Corollary 4.9, if $\left\{A_{Q}\right\}_{Q}$ is a doubling matrix sequence defined as follows.

Definition 1.3 (Doubling sequence). We say $\left\{A_{Q}\right\}_{Q} \in \mathcal{R} \mathcal{S}_{\mathcal{D}}$ is a (dyadic) doubling sequence (of order $p, 1 \leq p<\infty$ ) if there exists $\beta \geq n$ and $c \geq 1$ such that for all $P, Q$ dyadic

$$
\left\|A_{Q} A_{P}^{-1}\right\|^{p} \leq c \frac{|P|}{|Q|} \max \left(1,\left[\frac{l(Q)}{l(P)}\right]^{\beta}\right)\left(1+\frac{\operatorname{dist}(P, Q)}{\max (l(P), l(Q))}\right)^{\beta},
$$

where $|Q|$ is the Lebesgue measure of $Q$ and the norm on the left side is the operator (matrix) norm. 
Observe that if (1) holds for some $p$, then it holds for $1 \leq q<p$, since the right-hand side is $\geq 1$.

Our main goal is to identify the dual space of $\dot{B}_{p}^{\alpha q}(W)$. For $W \in A_{p}$ the result can be expressed in terms of matrix weights. However, even for $W \notin A_{p}$ but satisfying (A2) or (A3), we are able to characterize $\left[\dot{B}_{p}^{\alpha q}(W)\right]^{*}$ in terms of reducing operators. Set $1 / p+1 / p^{\prime}=1$ if $1<p<\infty$ and $p^{\prime}=\infty$ if $p=1 ; 1 / q+1 / q^{\prime}=1$ if $1<q<\infty$ and $q^{\prime}=\infty$ if $0<q \leq 1$. It is important to emphasize our convention for the duality pairing. In what follows, we say that a function space $Y$ is a dual of a function space $X$, and write $X^{*} \approx Y$, in the sense that each $y \in Y$ defines an element $l_{y}$ of $X^{*}$ via the pairing $l_{y}(x)=(x, y)=\int_{\mathbb{R}^{n}}\langle x(t), y(t)\rangle d t$ and every element of $X^{*}$ is of the kind $l_{y}$ for some $y \in Y$ with $\left\|l_{y}\right\| \approx\|y\|_{Y}$. (For example, $\left[L^{p}(W)\right]^{*} \approx L^{p^{\prime}}\left(W^{-p^{\prime} / p}\right)$, $1<p<\infty$, with the pairing $(\vec{f}, \vec{g})=\int_{\mathbb{R}^{n}}\langle\vec{f}(t), \vec{g}(t)\rangle_{\mathcal{H}} d t$; refer to Section 3 for more details.)

Theorem A1. Let $\alpha \in \mathbb{R}, 1 \leq p<\infty, 0<q<\infty$ and let $\left\{A_{Q}\right\}_{Q}$ be reducing operators of a matrix weight $W$.

(3) If $W$ satisfies any of $(\mathrm{A} 1)-(\mathrm{A} 3)$, then $\left[\dot{B}_{p}^{\alpha q}(W)\right]^{*} \approx \dot{B}_{p^{\prime}}^{-\alpha q^{\prime}}\left(\left\{A_{Q}^{-1}\right\}\right)$.

(For the proof refer to Section 5.)

Our next result identifies the dual space of the sequence (discrete) Besov space $\dot{b}_{p}^{\alpha q}(W)$. The connection between $\dot{b}_{p}^{\alpha q}(W)$ and $\dot{B}_{p}^{\alpha q}(W)$ is that $\vec{f} \in$ $\dot{B}_{p}^{\alpha q}(W)$ if and only if the appropriate wavelet coefficient sequence of $\vec{f}$ belongs to $\dot{b}_{p}^{\alpha q}(W)$ (see [5] for details). Recall the definitions of $\dot{b}_{p}^{\alpha q}(W)$ and $\dot{b}_{p}^{\alpha q}\left(\left\{A_{Q}\right\}\right)$ from $[5]$ :

Definition 1.4 (Matrix-weighted sequence Besov space $\dot{b}_{p}^{\alpha q}(W)$ ). For $\alpha \in \mathbb{R}, 1 \leq p<\infty, 0<q \leq \infty$ and $W$ a matrix weight, the space $\dot{b}_{p}^{\alpha q}(W)$ consists of all vector-valued sequences $\vec{s}=\left\{\vec{s}_{Q}\right\}_{Q \in \mathcal{D}}$, where $\vec{s}_{Q}=$ $\left(s_{Q}^{(1)}, \ldots, s_{Q}^{(m)}\right)^{\mathrm{T}}$, such that

$$
\begin{aligned}
& \left\|\left\{\vec{s}_{Q}\right\}_{Q}\right\|_{\dot{b}_{p}^{\alpha q}(W)} \\
& \quad=\left\|\left\{2^{\nu \alpha}\left\|\sum_{l(Q)=2^{-\nu}}|Q|^{-1 / 2}\left(\left\|W^{1 / p}(t) \vec{s}_{Q}\right\|_{\mathcal{H}}\right) \chi_{Q}(t)\right\|_{L^{p}(d t)}\right\}_{\nu}\right\|_{l^{q}}<\infty .
\end{aligned}
$$

Definition 1.5 (Averaging matrix-weighted discrete Besov space $\left.\dot{b}_{p}^{\alpha q}\left(\left\{A_{Q}\right\}\right)\right)$. For $\alpha \in \mathbb{R}, 1 \leq p \leq \infty, 0<q \leq \infty$ and $\left\{A_{Q}\right\}_{Q} \in \mathcal{R S}_{\mathcal{D}}$, the space $\dot{b}_{p}^{\alpha q}\left(\left\{A_{Q}\right\}\right)$ consists of all vector-valued sequences $\left\{\vec{s}_{Q}\right\}_{Q \in \mathcal{D}}$ such that 


$$
\begin{aligned}
\left\|\left\{\vec{s}_{Q}\right\}_{Q}\right\|_{\dot{b}_{p}^{\alpha q}\left(\left\{A_{Q}\right\}\right)} & =\left\|\left\{2^{\nu \alpha}\left\|\sum_{l(Q)=2^{-\nu}}|Q|^{-1 / 2}\left(\left\|A_{Q} \vec{s}_{Q}\right\|_{\mathcal{H}}\right) \chi_{Q}(t)\right\|_{L^{p}(d t)}\right\}_{\nu}\right\|_{l^{q}} \\
& =\left\|\left\{A_{Q} \vec{s}_{Q}\right\}_{Q}\right\|_{\dot{b}_{p}^{\alpha q}}<\infty .
\end{aligned}
$$

If $\left\{A_{Q}\right\}_{Q}$ is a sequence of reducing operators for a matrix weight $W$, then the norm equivalence

$$
\|\vec{s}\|_{\dot{b}_{p}^{\alpha q}(W)} \approx\|\vec{s}\|_{\dot{b}_{p}^{\alpha q}\left(\left\{A_{Q}\right\}\right)}
$$

holds for any matrix weight $W, \alpha \in \mathbb{R}, 0<q \leq \infty$ and $1 \leq p<\infty$ ([5, Lemma 7.1]). To make notation short, we will write $\dot{b}_{p}^{\alpha q}(W) \approx \dot{b}_{p}^{\alpha q}\left(\left\{A_{Q}\right\}\right)$ for the norm equivalence.

Theorem A2. Let $\alpha \in \mathbb{R}, 0<q<\infty, 1 \leq p<\infty$ and let $\left\{A_{Q}\right\}_{Q}$ be reducing operators of a matrix weight $W$. Then

$$
\left[\dot{b}_{p}^{\alpha q}(W)\right]^{*} \approx \dot{b}_{p^{\prime}}^{-\alpha q^{\prime}}\left(\left\{A_{Q}^{-1}\right\}\right) .
$$

Moreover, if $W \in A_{p}, 1<p<\infty$, then

$$
\left[\dot{b}_{p}^{\alpha q}(W)\right]^{*} \approx \dot{b}_{p^{\prime}}^{-\alpha q^{\prime}}\left(W^{-p^{\prime} / p}\right) .
$$

The paper is organized as follows. In Section 3 we discuss the discrete Besov space $\dot{b}_{p}^{\alpha q}(W)$. We use a "one at a time reduction" approach meaning we reduce the space $\dot{b}_{p}^{\alpha q}(W)$ in the following order:

$$
\dot{b}_{p}^{\alpha q}(W) \rightarrow \dot{b}_{p}^{\alpha q}\left(\left\{A_{Q}\right\}\right) \rightarrow \dot{b}_{p}^{\alpha q}\left(\mathbb{R}^{m}\right) \rightarrow \dot{b}_{p}^{\alpha q}\left(\mathbb{R}^{1}\right),
$$

where the last two spaces are unweighted vector-valued and scalar-valued discrete Besov spaces, and then identify the duals in the opposite order. A similar approach is used for $\dot{B}_{p}^{\alpha q}(W)$.

The fact that each $A_{Q}$ is constant on each dyadic cube $Q$ allows us to establish

$$
\left[\dot{b}_{p}^{\alpha q}\left(\left\{A_{Q}\right\}\right)\right]^{*} \approx \dot{b}_{p^{\prime}}^{-\alpha q^{\prime}}\left(\left\{A_{Q}^{-1}\right\}\right)
$$

for any $\left\{A_{Q}\right\}_{Q} \in \mathcal{R} \mathcal{S}_{\mathcal{D}}, \alpha \in \mathbb{R}, 0<q<\infty, 1 \leq p<\infty$. If the $A_{Q}$ 's are generated by a matrix weight $W$, then combining (4) and (7), we get (5) of Theorem A2.

In order to connect $\dot{b}_{p^{\prime}}^{-\alpha q^{\prime}}\left(\left\{A_{Q}^{-1}\right\}\right)$ with $\dot{b}_{p^{\prime}}^{-\alpha q^{\prime}}\left(\left\{A_{Q}^{\#}\right\}\right) \approx \dot{b}_{p^{\prime}}^{-\alpha q^{\prime}}\left(W^{-p^{\prime} / p}\right)$ (for the definition of $A_{Q}^{\#}$ refer to Section 2) the matrix $A_{p}$ condition is needed, though only for one direction of the embedding; the other direction is automatic. Thus, the following chain of the equivalences holds for $\dot{b}_{p}^{\alpha q}(W)$ :

$$
\begin{aligned}
{\left[\dot{b}_{p}^{\alpha q}(W)\right]^{*} } & \stackrel{\text { any } W}{\approx}\left[\dot{b}_{p}^{\alpha q}\left(\left\{A_{Q}\right\}\right)\right]^{*} \approx \dot{b}_{p^{\prime}}^{-\alpha q^{\prime}}\left(\left\{A_{Q}^{-1}\right\}\right) \\
& \approx \dot{A}_{p} \\
& \dot{b}_{p^{\prime}}^{-\alpha q^{\prime}}\left(\left\{A_{Q}^{\#}\right\}\right) \stackrel{\text { any } W}{\approx} \dot{b}_{p^{\prime}}^{-\alpha q^{\prime}}\left(W^{-p^{\prime} / p}\right) .
\end{aligned}
$$

This completes the proof of Theorem A2. 
In Section 4 we prove the norm equivalence between $\dot{B}_{p}^{\alpha q}\left(\left\{A_{Q}\right\}\right)$ and $\dot{b}_{p}^{\alpha q}\left(\left\{A_{Q}\right\}\right)$ for any doubling sequence $\left\{A_{Q}\right\}_{Q}$. Note that if the $A_{Q}$ 's are generated by a matrix weight $W$, then all that is required from the weight is the doubling condition. (Compare this with conditions (A1)-(A3) from [5] for the norm equivalence between the original matrix-weighted Besov spaces.)

For

$$
Q=Q_{\nu k}=\prod_{i=1}^{n}\left[\frac{k_{i}}{2^{\nu}}, \frac{k_{i}+1}{2^{\nu}}\right],
$$

$\nu \in \mathbb{Z}$ and $k \in \mathbb{Z}^{n}$, define $\varphi_{Q}(x)=|Q|^{-1 / 2} \varphi\left(2^{\nu} x-k\right)=|Q|^{1 / 2} \varphi_{\nu}\left(x-x_{Q}\right)$, where $x_{Q}=2^{-\nu} k$ is the lower left corner of $Q_{\nu k}$.

Theorem 1.6. Let $\alpha \in \mathbb{R}, 0<q \leq \infty, 1 \leq p<\infty$ and $\left\{A_{Q}\right\}_{Q}$ be a doubling sequence (of order $p$ ). Then for $\vec{s}_{Q}(\vec{f})=\left\langle\vec{f}, \varphi_{Q}\right\rangle$,

$$
\|\vec{f}\|_{\dot{B}_{p}^{\alpha q}\left(\left\{A_{Q}\right\}\right)} \approx\left\|\left\{\vec{s}_{Q}(\vec{f})\right\}_{Q}\right\|_{\dot{b}_{p}^{\alpha q}\left(\left\{A_{Q}\right\}\right)} .
$$

In Section 5 we establish the correspondence between the continuous Besov spaces $\dot{B}_{p}^{\alpha q}(W)$ and $\dot{B}_{p}^{\alpha q}\left(\left\{A_{Q}\right\}\right)$.

Lemma 1.7. Let $\alpha \in \mathbb{R}, 0<q \leq \infty$ and $1 \leq p<\infty$. If $W$ satisfies any of (A1)-(A3) and $\left\{A_{Q}\right\}_{Q}$ is a sequence of reducing operators generated by $W$, then

$$
\dot{B}_{p}^{\alpha q}(W) \approx \dot{B}_{p}^{\alpha q}\left(\left\{A_{Q}\right\}\right) .
$$

For one direction of the above equivalence it suffices to have $W$ doubling.

In Section 6 it is shown that if $\left\{A_{Q}\right\}_{Q}$ is a doubling sequence of order $p$, $1 \leq p<\infty$, then

$$
\left[\dot{B}_{p}^{\alpha q}\left(\left\{A_{Q}\right\}\right)\right]^{*} \approx \dot{B}_{p^{\prime}}^{-\alpha q^{\prime}}\left(\left\{A_{Q}^{-1}\right\}\right) .
$$

Using the above duality and equivalence, we get the following chain:

$$
\begin{aligned}
{\left[\dot{B}_{p}^{\alpha q}(W)\right]^{*} } & \stackrel{(1)}{\approx}\left[\dot{B}_{p}^{\alpha q}\left(\left\{A_{Q}\right\}\right)\right]^{*} \approx \dot{B}_{p^{\prime}}^{-\alpha q^{\prime}}\left(\left\{A_{Q}^{-1}\right\}\right) \\
& \stackrel{A_{p}}{\approx} \dot{B}_{p^{\prime}}^{-\alpha q^{\prime}}\left(\left\{A_{Q}^{\#}\right\}\right) \stackrel{(4)}{\approx} \dot{B}_{p^{\prime}}^{-\alpha q^{\prime}}\left(W^{-p^{\prime} / p}\right),
\end{aligned}
$$

where the equivalence (1) holds if $W$ satisfies any of (A1)-(A3) and (4) holds if $W^{-p^{\prime} / p}$ satisfies any of (A1)-(A3) properly adjusted (see Section 2). The third equivalence holds under the $A_{p}$ condition. However, the $A_{p}$ condition is only needed for one direction of the embedding. This proves Theorem A1.

In the last section we consider inhomogeneous function spaces and transfer all the above theory to the inhomogeneous case. 
2. Definitions and notation. Given a matrix weight $W$, for each dyadic cube $Q$ in $\mathbb{R}^{n}$ consider a reducing operator $A_{Q}$ corresponding to the $L^{p}$ average over $Q$ of the norm $\left\|W^{1 / p} \cdot\right\|_{\mathcal{H}}$, i.e.,

$$
\left\|A_{Q} x\right\|_{\mathcal{H}} \approx\left(\frac{1}{|Q|} \int_{Q}\left\|W^{1 / p}(t) x\right\|_{\mathcal{H}}^{p} d t\right)^{1 / p} \quad \text { for all } x \in \mathcal{H} .
$$

Thus, we are dealing with a family of norms $\varrho_{t}(x)=\left\|W^{1 / p}(t) x\right\|_{\mathcal{H}}$. By definition, the dual norms are $\varrho_{t}^{*}(x)=\left\|W^{-1 / p}(t) x\right\|_{\mathcal{H}}$ and reducing operators for their $L^{p^{\prime}}$ averages over a cube $Q$ are

$$
\left\|A_{Q}^{\#} x\right\|_{\mathcal{H}} \approx\left(\frac{1}{|Q|} \int_{Q}\left\|W^{-1 / p}(t) x\right\|_{\mathcal{H}}^{p^{\prime}} d t\right)^{1 / p^{\prime}} .
$$

In other words, $\left\{A_{Q}^{\#}\right\}_{Q}$ is a reducing sequence for the "dual" matrix weight $W^{-p^{\prime} / p}$ (for more details refer to [10], [5]).

Recall that the matrix $A_{p}$ condition is $\left\|A_{Q}^{\#} A_{Q}\right\| \leq c$ for every cube $Q \subseteq \mathbb{R}^{n}$, and the opposite inequality $\left\|\left(A_{Q}^{\#} A_{Q}\right)^{-1}\right\| \leq c$ always holds as a simple consequence of Hölder's inequality: for any $x, y \in \mathcal{H}$ we have

$$
\begin{aligned}
|(x, y)| & \leq\left(\int_{Q}\left\|W^{1 / p}(t) x\right\|^{p} \frac{d t}{|Q|}\right)^{1 / p}\left(\int_{Q}\left\|W^{-1 / p}(t) y\right\|^{p^{\prime}} \frac{d t}{|Q|}\right)^{1 / p^{\prime}} \\
& \approx\left\|A_{Q} x\right\|\left\|A_{Q}^{\#} y\right\|,
\end{aligned}
$$

which implies $\left\|A_{Q} x\right\| \geq c\left\|\left(A_{Q}^{\#}\right)^{-1} x\right\|$ for any $x \in \mathcal{H}$ and, thus, the above statement follows.

A condition which is weaker than $A_{p}$ for a matrix weight $W$ is the doubling condition:

Definition 2.1 (Doubling matrix). A matrix weight $W: \mathbb{R}^{n} \rightarrow \mathcal{M}$ is called a doubling matrix (of order $p, 1 \leq p<\infty$ ), if there exists a constant $c=c_{p, n}$ such that for any $x \in \mathcal{H}$, any $\delta>0$ and any $z \in \mathbb{R}^{n}$,

$$
\int_{B_{2 \delta}(z)}\left\|W^{1 / p}(t) x\right\|_{\mathcal{H}}^{p} d t \leq c \int_{B_{\delta}(z)}\left\|W^{1 / p}(t) x\right\|_{\mathcal{H}}^{p} d t
$$

i.e., the scalar measure $w_{x}(t)=\left\|W^{1 / p}(t) x\right\|_{\mathcal{H}}^{p}$ is uniformly doubling and not identically zero (a.e.). If $c=2^{\beta}$ is the smallest constant for which (11) holds, then $\beta$ is called the doubling exponent of $W$.

Observe that if $W$ is a doubling matrix weight (of order $p$ ), then $\left\{A_{Q}\right\}_{Q}$ is a doubling sequence (of order $p$ ). The fact that $A_{p}$ implies doubling in the scalar case is quite straightforward and can be found in [7]. The vector case can be found in [6]. Also note that $\beta \geq n$ and for the Lebesgue measure $\beta=n$. 
By saying $W^{-p^{\prime} / p}$ satisfies any of (A1)-(A3), we mean either $W^{-p^{\prime} / p} \in$ $A_{p^{\prime}}$ with $1<p^{\prime}<\infty$ (which is equivalent to $W \in A_{p}, 1<p<\infty$ ), or $W^{-p^{\prime} / p}$ is a doubling matrix of order $p^{\prime}$ with $p^{\prime}>\beta^{*}$, where $\beta^{*}$ is the doubling exponent of $W^{-p^{\prime} / p}$, or $W^{-p^{\prime} / p}$ is a diagonal doubling matrix of order $p^{\prime}$ with $1<p^{\prime}<\infty$.

In order to establish the connection between matrix weighted Besov spaces and averaging Besov spaces, we use an auxiliary $L^{p}$ space:

Definition 2.2 (Averaging space $L^{p}\left(\left\{A_{Q}\right\}, \nu\right)$ ). For $\nu \in \mathbb{Z}, 1 \leq p \leq \infty$ and $\left\{A_{Q}\right\}_{Q} \in \mathcal{R} \mathcal{S}_{\mathcal{D}}$, the space $L^{p}\left(\left\{A_{Q}\right\}, \nu\right)$ consists of all vector-valued locally integrable functions $\vec{f}$ such that

$$
\|\vec{f}\|_{L^{p}\left(\left\{A_{Q}\right\}, \nu\right)}=\left\|\sum_{l(Q)=2^{-\nu}} \chi_{Q}(t) A_{Q} \vec{f}(t)\right\|_{L^{p}(d t)}<\infty .
$$

Note that $\|\vec{f}\|_{\dot{B}_{p}^{\alpha q}\left(\left\{A_{Q}\right\}\right)}=\left\|\left\{2^{\nu \alpha}\left\|\varphi_{\nu} * \vec{f}\right\|_{L^{p}\left(\left\{A_{Q}\right\}, \nu\right)}\right\}_{\nu}\right\|_{l^{q}}$. To make notation short, define $Q_{\nu}=\left\{Q \in \mathcal{D}: l(Q)=2^{-\nu}\right\}$.

3. Duality of sequence Besov spaces. An important tool that we need is the duality on $l^{q}(X)$ with $X$ being a Banach space. By definition $l^{q}(X), 0<q<\infty$, is the set of all sequences $\left\{f_{\nu}\right\}_{\nu \in \mathbb{Z}}$ with $f_{\nu} \in X, \nu \in \mathbb{Z}$ such that $\left(\sum_{\nu \in \mathbb{Z}}\left\|f_{\nu}\right\|_{X}^{q}\right)^{1 / q}<\infty$. If $1 \leq q<\infty$, then $\left(l^{q}(X)\right)^{*}=l^{q^{\prime}}\left(X^{*}\right)$ (see [1, Chapter 8]), and if $g$ is a continuous linear functional on $l^{q}(X)$ identified with $\left\{g_{\nu}\right\}_{\nu \in \mathbb{Z}} \in l^{q^{\prime}}\left(X^{*}\right)$, then the duality is represented as

$$
g(f)=(f, g)=\sum_{\nu \in \mathbb{Z}}\left\langle f_{\nu}, g_{\nu}\right\rangle_{X},
$$

where $\left\langle f_{\nu}, g_{\nu}\right\rangle_{X}=g_{\nu}\left(f_{\nu}\right)$ is the pairing between $X$ and $X^{*}$. We will mainly be concerned with $X=L^{p}, 1 \leq p<\infty$, or $L^{p}(W), 1<p<\infty$, and, thus, $X^{*}=L^{p^{\prime}}$ or $L^{p^{\prime}}\left(W^{-p^{\prime} / p}\right)$, respectively, with the pairing $\langle f, g\rangle_{X}=$ $\int\langle f(x), g(x)\rangle_{\mathcal{H}} d x$.

If $0<q<1$, and $X=L^{p}, 1 \leq p<\infty$, then $\left(l^{q}\left(L^{p}\right)\right)^{*}=l^{\infty}\left(L^{p^{\prime}}\right)$ (see $[9$, p. 177]) and the pairing is defined as above.

Theorem 3.1. Let $W$ be a matrix weight, $\alpha \in \mathbb{R}, 0<q<\infty, 1<p$ $<\infty$. Then

(i) $\dot{b}_{p^{\prime}}^{-\alpha q^{\prime}}\left(W^{-p^{\prime} / p}\right) \subseteq\left[\dot{b}_{p}^{\alpha q}(W)\right]^{*}$ always,

(ii) $\left[\dot{b}_{p}^{\alpha q}(W)\right]^{*} \subseteq \dot{b}_{p^{\prime}}^{-\alpha q^{\prime}}\left(W^{-p^{\prime} / p}\right)$ if $W \in A_{p}$.

We will prove this theorem, which implies (6) of Theorem A2, in several steps. The use of reducing operators is essential and helps to understand why certain conditions on the weight $W$ are necessary. 
Proof of (i) of Theorem 3.1. For each $\vec{t} \in \dot{b}_{p^{\prime}}^{-\alpha q^{\prime}}\left(W^{-p^{\prime} / p}\right)$ define a functional $l_{\vec{t}}$ on $\dot{b}_{p}^{\alpha q}(W)$ by

$$
l_{\vec{t}}(\vec{s})=(\vec{s}, \vec{t})=\sum_{Q}\left\langle\vec{s}_{Q}, \vec{t}_{Q}\right\rangle_{\mathcal{H}} \quad \text { for any } \vec{s}=\left\{\vec{s}_{Q}\right\}_{Q} \in \dot{b}_{p}^{\alpha q}(W) .
$$

The calculations below show that this sum converges and $l_{\vec{t}} \in\left[\dot{b}_{p}^{\alpha q}(W)\right]^{*}$ :

$$
\begin{aligned}
& \left|\sum_{Q}\left\langle\vec{s}_{Q}, \vec{t}_{Q}\right\rangle_{\mathcal{H}}\right| \\
& \quad \leq \sum_{\nu \in \mathbb{Z}} \int_{\mathbb{R}^{n}} \sum_{Q \in Q_{\nu}}|Q|^{-1}\left|\left\langle W^{-1 / p}(x) W^{1 / p}(x) \vec{s}_{Q}, \vec{t}_{Q}\right\rangle_{\mathcal{H}}\right| \chi_{Q}(x) d x .
\end{aligned}
$$

Using the self-adjointness of $W$ and the Cauchy-Schwarz inequality, we bound (12) by

$$
\begin{aligned}
\sum_{\nu \in \mathbb{Z}} \int_{\mathbb{R}^{n}} \sum_{Q \in Q_{\nu}}\left(|Q|^{-\alpha / n-1 / 2} \chi_{Q}(x)\left\|W^{1 / p}(x) \vec{s}_{Q}\right\|_{\mathcal{H}}\right) & \\
& \times\left(|Q|^{\alpha / n-1 / 2} \chi_{Q}(x)\left\|W^{-1 / p}(x) \vec{t}_{Q}\right\|_{\mathcal{H}}\right) d x .
\end{aligned}
$$

Applying Hölder's inequality several times, we estimate $l_{\vec{t}}(\vec{s})$ by

$$
\begin{aligned}
&\left.\sum_{\nu \in \mathbb{Z} \mathbb{R}^{n}} \int_{Q \in Q_{\nu}}\left(|Q|^{-\alpha / n-1 / 2} \chi_{Q}(x)\left\|W^{1 / p}(x) \vec{s}_{Q}\right\|_{\mathcal{H}}\right)^{p}\right)^{1 / p} \\
& \times\left(\sum_{Q \in Q_{\nu}}\left(|Q|^{\alpha / n-1 / 2} \chi_{Q}(x)\left\|W^{-1 / p}(x) \vec{t}_{Q}\right\|_{\mathcal{H}}\right)^{p^{\prime}}\right)^{1 / p^{\prime}} d x \\
& \leq \sum_{\nu \in \mathbb{Z}}\left\|\sum_{Q \in Q_{\nu}}|Q|^{-\alpha / n-1 / 2} \chi_{Q} \vec{s}_{Q}\right\|_{L^{p}(W)}\left\|\sum_{Q \in Q_{\nu}}|Q|^{\alpha / n-1 / 2} \chi_{Q} \vec{t}_{Q}\right\|_{L^{p^{\prime}}\left(W^{-p^{\prime} / p}\right)} \\
& \leq\|\vec{s}\|_{\dot{b}_{p}^{\alpha q}(W)}\|\vec{t}\|_{\dot{b}_{p^{\prime}}^{-\alpha q^{\prime}}\left(W^{\left.-p^{\prime} / p\right)}\right.},
\end{aligned}
$$

for $1<q<\infty$. In the case of $0<q \leq 1$, we bound (13) by

$$
\|\vec{s}\|_{\dot{b}_{p}^{\alpha 1}(W)}\|\vec{t}\|_{\dot{b}_{p^{\prime}}^{-\alpha \infty}\left(W^{\left.-p^{\prime} / p\right)}\right.} .
$$

Since $l^{q}$ is embedded into $l^{1}$ when $0<q \leq 1$, we estimate the previous product by $\|\vec{s}\|_{\dot{b}_{p}^{\alpha q}(W)}\|\vec{t}\|_{\dot{b}_{p^{\prime}}^{-\alpha \infty}\left(W^{\left.-p^{\prime} / p\right)}\right.}$.

In terms of reducing operators (or using (4)) the previous lemma states

$$
\dot{b}_{p^{\prime}}^{-\alpha q^{\prime}}\left(\left\{A_{Q}^{\#}\right\}\right) \subseteq\left[\dot{b}_{p}^{\alpha q}\left(\left\{A_{Q}\right\}\right)\right]^{*} .
$$

If we follow along the lines of the proof again but instead of $W^{-1 / p}(t) W^{1 / p}(t)$ in (12) use $A_{Q}^{-1} A_{Q}$, then we obtain the following statement. 
Lemma 3.2. Let $\alpha \in \mathbb{R}, 0<q<\infty, 1 \leq p<\infty$ and $\left\{A_{Q}\right\}_{Q} \in \mathcal{R S}_{\mathcal{D}}$. Then

$$
\dot{b}_{p^{\prime}}^{-\alpha q^{\prime}}\left(\left\{A_{Q}^{-1}\right\}\right) \subseteq\left[\dot{b}_{p}^{\alpha q}\left(\left\{A_{Q}\right\}\right)\right]^{*} .
$$

In fact, if only we have proven (15), then (14) (and equivalently part (i) of Theorem 3.1) could have been obtained as a consequence of (15) and [5, Corollary 7.4], i.e.,

$$
\dot{b}_{p^{\prime}}^{-\alpha q^{\prime}}\left(\left\{A_{Q}^{\#}\right\}\right) \subseteq \dot{b}_{p^{\prime}}^{-\alpha q^{\prime}}\left(\left\{A_{Q}^{-1}\right\}\right) \subseteq\left[\dot{b}_{p}^{\alpha q}\left(\left\{A_{Q}\right\}\right)\right]^{*} .
$$

Observe that (15) holds for any $\left\{A_{Q}\right\}_{Q} \in \mathcal{R} \mathcal{S}_{\mathcal{D}}$, not necessarily generated by $W$.

Now we will study the opposite embeddings. By Lemma 3.3 below, we will get

$$
\left[\dot{b}_{p}^{\alpha q}\left(\left\{A_{Q}\right\}\right)\right]^{*} \subseteq \dot{b}_{p^{\prime}}^{-\alpha q^{\prime}}\left(\left\{A_{Q}^{-1}\right\}\right)
$$

without any additional assumptions on the sequence $\left\{A_{Q}\right\}_{Q}$. Note that combining (15) and (17), we obtain (7). If we apply [5, Corollary 7.4] again, (17) is continued as

$$
\left[\dot{b}_{p}^{\alpha q}\left(\left\{A_{Q}\right\}\right)\right]^{*} \subseteq \dot{b}_{p^{\prime}}^{-\alpha q^{\prime}}\left(\left\{A_{Q}^{-1}\right\}\right) \stackrel{A_{p}}{\subseteq} \dot{b}_{p^{\prime}}^{-\alpha q^{\prime}}\left(\left\{A_{Q}^{\#}\right\}\right) \approx \dot{b}_{p^{\prime}}^{-\alpha q^{\prime}}\left(W^{-p^{\prime} / p}\right)
$$

with the second embedding being held under the $A_{p}$ condition. Thus, the embedding (ii) of Theorem 3.1 holds if $W \in A_{p}$.

Lemma 3.3. Let $\left\{A_{Q}\right\}_{Q} \in \mathcal{R S}_{\mathcal{D}}, \alpha \in \mathbb{R}, 0<q<\infty, 1 \leq p<\infty$. Then (17) holds.

Proof. Let $l \in\left[\dot{b}_{p}^{\alpha q}\left(\left\{A_{Q}\right\}\right)\right]^{*}$. We show that there exists $\vec{t} \in \dot{b}_{p^{\prime}}^{-\alpha q^{\prime}}\left(\left\{A_{Q}^{-1}\right\}\right)$ such that for any $\vec{s} \in \dot{b}_{p}^{\alpha q}\left(\left\{A_{Q}\right\}\right)$,

$$
l(\vec{s})=(\vec{s}, \vec{t})=\sum_{Q}\left\langle\vec{s}_{Q}, \vec{t}_{Q}\right\rangle_{\mathcal{H}} \quad \text { and } \quad\|\vec{t}\|_{\dot{b}_{p^{\prime}}^{-\alpha q^{\prime}}\left(\left\{A_{Q}^{-1}\right\}\right)} \leq\|l\| .
$$

Let $\vec{e}_{J}^{(k)}$ denote a vector-valued sequence enumerated by dyadic cubes such that in the $k$ th component ( $k$ th row) of this vector the $J$ th entry (corresponding to the dyadic cube $J$ ) is equal to 1 and all other entries are zero: $\vec{e}_{J}^{(k)}=\left(\ldots,\{0\}_{Q}, \ldots,\left\{\ldots 0 \ldots 1_{J \text { th entry }} \ldots 0 \ldots\right\}_{Q}(k \text { th row }), \ldots,\{0\}_{Q}, \ldots\right)^{\mathrm{T}}$.

Now if $\vec{s}$ has only finitely many non-zero entries, i.e.,

$$
\vec{s}=\sum_{\{Q\} \text { finite }} \sum_{k=1}^{m} s_{Q}^{(k)} \vec{e}_{Q}^{(k)},
$$

then by linearity

$$
l(\vec{s})=\sum_{\{Q\} \text { finite }} \sum_{k=1}^{m} s_{Q}^{(k)} l\left(\vec{e}_{Q}^{(k)}\right)=: \sum_{\{Q\} \text { finite }} \sum_{k=1}^{m} s_{Q}^{(k)} t_{Q}^{(k)} .
$$


By continuity, since finitely non-zero sequences are dense $(p, q<\infty)$, we get

$$
l(\vec{s})=\sum_{Q \in \mathcal{D}} \sum_{k=1}^{m} s_{Q}^{(k)} t_{Q}^{(k)}=\sum_{Q \in \mathcal{D}}\left\langle\vec{s}_{Q}, \vec{t}_{Q}\right\rangle_{\mathcal{H}} \quad \text { for any } \vec{s} \in \dot{b}_{p}^{\alpha q}\left(\left\{A_{Q}\right\}\right) .
$$

Now everything is set up to show that $\vec{t}:=\left(\left\{t_{Q}^{(1)}\right\}_{Q}, \ldots,\left\{t_{Q}^{(m)}\right\}_{Q}\right)^{\mathrm{T}} \in$ $\dot{b}_{p^{\prime}}^{-\alpha q^{\prime}}\left(\left\{A_{Q}^{-1}\right\}\right)$. For $\overrightarrow{\widetilde{s}} \in \dot{b}_{p}^{\alpha q}\left(\mathbb{R}^{m}\right)$, set $\vec{s}_{Q}=A_{Q}^{-1} \overrightarrow{\widetilde{s}}$ and define

$$
\begin{aligned}
\widetilde{l}(\overrightarrow{\widetilde{s}}) & :=l\left(\left\{A_{Q}^{-1} \overrightarrow{\widetilde{s}}_{Q}\right\}_{Q}\right)=l\left(\left\{\vec{s}_{Q}\right\}_{Q}\right)=\sum_{Q}\left\langle\vec{s}_{Q}, \vec{t}_{Q}\right\rangle_{\mathcal{H}} \\
& =\sum_{Q}\left\langle A_{Q} \vec{s}_{Q}, A_{Q}^{-1} \vec{t}_{Q}\right\rangle_{\mathcal{H}}=\sum_{Q}\left\langle\overrightarrow{\widetilde{s}}_{Q}, \overrightarrow{\widetilde{t}}_{Q}\right\rangle_{\mathcal{H}},
\end{aligned}
$$

where $\overrightarrow{\widetilde{t}}_{Q}=A_{Q}^{-1} \vec{t}_{Q}$. By the above,

$$
|\widetilde{l}(\overrightarrow{\widetilde{s}})| \leq c\left\|\left\{\vec{s}_{Q}\right\}_{Q}\right\|_{\dot{b}_{p}^{\alpha q}\left(\left\{A_{Q}\right\}\right)}=c\left\|\left\{\overrightarrow{\widetilde{s}}_{Q}\right\}_{Q}\right\|_{\dot{b}_{p}^{\alpha q}\left(\mathbb{R}^{m}\right)},
$$

i.e., $l$ induces a continuous linear functional $\widetilde{l}$ on $\dot{b}_{p}^{\alpha q}\left(\mathbb{R}^{m}\right)$. By Lemma 3.4 below, $\left\{\overrightarrow{\widetilde{t}}_{Q}\right\}_{Q} \in \dot{b}_{p^{\prime}}^{-\alpha q^{\prime}}\left(\mathbb{R}^{m}\right)$. Since the inside $L^{p^{\prime}}$-norm of the $\dot{b}_{p^{\prime}}^{-\alpha q^{\prime}}\left(\mathbb{R}^{m}\right)$-norm of $\overrightarrow{\widetilde{t}}$ is

$$
\begin{aligned}
\left\|\sum_{Q \in Q_{\nu}}|Q|^{-1 / 2} \overrightarrow{\widetilde{t}}_{Q} \chi_{Q}\right\|_{L^{p^{\prime}}} & =\left\|\sum_{Q \in Q_{\nu}}|Q|^{-1 / 2}\right\| A_{Q}^{-1} \vec{t}_{Q}\left\|_{\mathcal{H}} \chi_{Q}\right\|_{L^{p^{\prime}}} \\
& =\left\|\sum_{Q \in Q_{\nu}}|Q|^{-1 / 2} \vec{t}_{Q} \chi_{Q}\right\|_{L^{p^{\prime}}\left(\left\{A_{Q}^{-1}\right\}\right)}
\end{aligned}
$$

$\vec{t} \in \dot{b}_{p^{\prime}}^{-\alpha q^{\prime}}\left(\left\{A_{Q}^{-1}\right\}\right)$ and the lemma is proved.

Lemma 3.4. Let $\alpha \in \mathbb{R}, 0<q<\infty, 1 \leq p<\infty$. Then

$$
\left[\dot{b}_{p}^{\alpha q}\left(\mathbb{R}^{m}\right)\right]^{*} \approx \dot{b}_{p^{\prime}}^{-\alpha q^{\prime}}\left(\mathbb{R}^{m}\right) .
$$

Proof. It only suffices to show the scalar case $(m=1)$ of $(18)$, since $\vec{s} \in \dot{b}_{p}^{\alpha q}\left(\mathbb{R}^{m}\right)$ means that each component $s^{(i)}$ belongs to $\dot{b}_{p}^{\alpha q}$ and by making zero all but one of the components of an arbitrary $\vec{s}$ we obtain (18).

The embedding $\left[\dot{b}_{p}^{\alpha q}\right]^{*} \supseteq \dot{b}_{p^{\prime}}^{-\alpha q^{\prime}}$ is a trivial application of Hölder's inequality plus the embedding $\dot{b}_{p}^{\alpha q} \rightarrow \dot{b}_{p}^{\alpha 1}$ for $q<1$, so we only concentrate on the opposite embedding.

Suppose $l \in\left[\dot{b}_{p}^{\alpha q}\right]^{*}$. Using linearity and continuity, $l$ can be represented by some sequence $\left\{t_{Q}\right\}_{Q}$ as $l(s)=\sum_{Q} s_{Q} \bar{t}_{Q}$ for any $s=\left\{s_{Q}\right\} \in \dot{b}_{p}^{\alpha q}$ and

$$
|l(s)|=\left|\sum_{Q} s_{Q} \bar{t}_{Q}\right| \leq\|l\|\|s\|_{\dot{b}_{p}^{\alpha q}} .
$$


CASE $q \geq 1$. For each $\nu \in \mathbb{Z}$ let $f_{\nu}(s)(x)=\sum_{Q \in Q_{\nu}}|Q|^{-\alpha / n-1 / 2} s_{Q} \chi_{Q}(x)$. Define a map $I: \dot{b}_{p}^{\alpha q} \rightarrow l^{q}\left(L^{p}\right)$ by $I(s)=\left\{f_{\nu}(s)\right\}_{\nu \in \mathbb{Z}}$. Observe that $\|I(s)\|_{l^{q}\left(L^{p}\right)}$ $=\|s\|_{\dot{b}_{p}^{\alpha q}}$, in other words, by the natural construction $I$ is a linear isometry onto the subspace $I\left(\dot{b}_{p}^{\alpha q}\right)$ of $l^{q}\left(L^{p}\right)$. Then $l$ induces a continuous linear functional $\widetilde{l}$ on $I\left(\dot{b}_{p}^{\alpha q}\right) \subseteq l^{q}\left(L^{p}\right)$ (continuous in $l^{q}\left(L^{p}\right)$-norm) by $\widetilde{l}(I(s))=l(s)$. Since $l^{q}\left(L^{p}\right)$ is a Banach space, by the Hahn-Banach Theorem $\widetilde{l}$ extends to a continuous linear functional $\widetilde{l}_{\text {ext }}$ on all of $l^{q}\left(L^{p}\right)$ with $\left\|\widetilde{l}_{\text {ext }}\right\|=\|\widetilde{l}\| \leq\|l\|$. Since $\left[l^{q}\left(L^{p}\right)\right]^{*}=l^{q^{\prime}}\left(L^{p^{\prime}}\right), \widetilde{l}_{\text {ext }}$ is represented by a sequence $g=\left\{g_{\nu}\right\}_{\nu \in \mathbb{Z}} \in$ $l^{q^{\prime}}\left(L^{p^{\prime}}\right)$ with $\|g\|=\left\|\left\{g_{\nu}\right\}_{\nu}\right\|_{l^{q^{\prime}}\left(L^{p^{\prime}}\right)} \leq\|l\|$ and

$$
\sum_{Q} s_{Q} \bar{t}_{Q}=l(s)=\widetilde{l}\left(\left\{f_{\nu}(s)\right\}\right)=\sum_{\nu \in \mathbb{Z}} \int_{\mathbb{R}^{n}} f_{\nu}(s)(x) \bar{g}_{\nu}(x) d x \quad \text { for any } \vec{s} \in \dot{b}_{p}^{\alpha q},
$$

or

$$
\sum_{Q} s_{Q} \bar{t}_{Q}=\sum_{\nu \in \mathbb{Z}} \sum_{Q \in Q_{\nu}}|Q|^{-\alpha / n-1 / 2} s_{Q} \int_{Q} \bar{g}_{\nu}(x) d x .
$$

Taking $s_{Q}=0$ for all but one cube, we get $t_{Q}=|Q|^{-\alpha / n+1 / 2}\left\langle g_{\nu}\right\rangle_{Q}$. Using Hölder's inequality, we have

$$
\|t\|_{\dot{b}_{p^{\prime}}^{-\alpha q^{\prime}}}=\left\|\left\{\left\|\sum_{Q \in Q_{\nu}}\left\langle g_{\nu}\right\rangle_{Q} \chi_{Q}\right\|_{L^{p^{\prime}}}\right\}_{\nu}\right\|_{l_{q^{\prime}}} \leq\left\|\left\{\vec{g}_{\nu}\right\}_{\nu}\right\|_{l^{q^{\prime}}\left(L^{p^{\prime}}\right)} \leq\|l\| .
$$

CASE $0<q<1$. Suppose $1<p<\infty$. Fix $\nu \in \mathbb{Z}$ and let $F_{\nu}$ denote a finite collection of cubes from $Q_{\nu}$. Set $\tau_{\nu}=\sum_{Q \in F_{\nu}}\left(|Q|^{\alpha / n-1 / 2+1 / p^{\prime}}\left|t_{Q}\right|\right)^{p^{\prime}}$. Since the sum is finite, $\tau_{\nu}<\infty$. Let $s_{Q}=|Q|^{\left(\alpha / n-1 / 2+1 / p^{\prime}\right) p^{\prime}}\left|t_{Q}\right|^{p^{\prime}-2} t_{Q}$ if $Q \in F_{\nu}$ and $t_{Q} \neq 0$; otherwise let $s_{Q}=0$. Note that $\left\|\left\{s_{Q}\right\}_{Q}\right\|_{\dot{b}_{p}^{\alpha q}}=\tau_{\nu}^{1 / p}$. Observe that $\sum_{Q} s_{Q} \bar{t}_{Q}=\tau_{\nu}$, and by (19), $\tau_{\nu} \leq\|l\|\|s\|_{\dot{b}_{p}^{\alpha q}}=\|l\| \tau_{\nu}^{1 / p}$. Since $\tau_{\nu}$ is finite, we get $\tau_{\nu}^{1 / p^{\prime}} \leq\|l\|$ and the estimate holds independently of the collection $F_{\nu}$ taken. Hence, we can pass to the limit from $F_{\nu}$ to $Q_{\nu}$. Then,

$$
\begin{aligned}
\|t\|_{\dot{b}_{p^{\prime}}^{-\alpha \infty}} & =\sup _{\nu \in \mathbb{Z}}\left(\sum_{Q \in Q_{\nu}}\left(|Q|^{\alpha / n-1 / 2+1 / p^{\prime}}\left|t_{Q}\right|\right)^{p^{\prime}}\right)^{1 / p^{\prime}} \\
& =\sup _{\nu \in \mathbb{Z}} \tau_{\nu}^{1 / p^{\prime}} \leq\|l\| \quad \text { or } \quad t \in \dot{b}_{p^{\prime}}^{-\alpha \infty} .
\end{aligned}
$$

Now assume $p=1$. Fix $P \in \mathcal{D}$ and define $s^{(P)}=\left\{s_{Q}^{(P)}\right\}_{Q}$ by $s_{Q}^{(P)}=$ $|Q|^{\alpha / n-1 / 2} \operatorname{sgn} \bar{t}_{Q}$ if $Q=P$ and $s_{Q}^{(P)}=0$ otherwise. Then $\left\|\left\{s_{Q}^{(P)}\right\}_{Q}\right\|_{\dot{b}_{1}^{\alpha q}}=1$ and $|P|^{\alpha / n-1 / 2}\left|t_{P}\right|=\sum_{Q} s_{Q}^{(P)} \bar{t}_{Q}=l\left(s^{(P)}\right) \leq\|l\|\left\|\left\{s_{Q}^{(P)}\right\}_{Q}\right\|_{\dot{b}_{1}^{\alpha q}}=\|l\|$ for any $P \in \mathcal{D}$. Hence,

$$
\|t\|_{\dot{b}_{\infty}^{-\alpha \infty}}=\sup _{P \in \mathcal{D}}|P|^{\alpha / n-1 / 2}\left|t_{P}\right| \leq\|l\| \quad \text { or } \quad t \in \dot{b}_{\infty}^{-\alpha \infty} .
$$




\section{Equivalence of sequence and discrete averaging Besov spaces.}

In this section we discuss norm equivalence between $\dot{B}_{p}^{\alpha q}\left(\left\{A_{Q}\right\}\right)$ and $\dot{b}_{p}^{\alpha q}\left(\left\{A_{Q}\right\}\right)$. We suppose $\alpha \in \mathbb{R}, 0<q \leq \infty$ and $1 \leq p<\infty$ for all statements in this section. If $q=\infty$, then set $q^{\prime}=1$.

Definition 4.1. For $\nu \in \mathbb{Z}$ let $E_{\nu}=\left\{\vec{f}: f_{i} \in \mathcal{S}^{\prime}\right.$ with supp $\widehat{f}_{i} \subseteq\{\xi \in$ $\left.\left.\mathbb{R}^{n}:|\xi| \leq 2^{\nu+1}\right\}, i=1, \ldots, m\right\}$.

The following decomposition of an exponential type function is a useful tool in studying the norm equivalence (for the proof the reader is referred to $[3$, p. 55$])$ :

Lemma 4.2. Suppose that $g \in \mathcal{S}^{\prime}\left(\mathbb{R}^{n}\right), h \in \mathcal{S}\left(\mathbb{R}^{n}\right)$ and $\operatorname{supp} \widehat{g}$, supp $\widehat{h} \subseteq$ $\left\{|\xi|<2^{\nu} \pi\right\}$ for some $\nu \in \mathbb{Z}$. Then

$$
(g * h)(x)=\sum_{k \in \mathbb{Z}^{n}} 2^{-\nu n} g\left(2^{-\nu} k\right) h\left(x-2^{-\nu} k\right) .
$$

Let $\Gamma=\left\{\gamma \in \mathcal{S}: \widehat{\gamma}=1\right.$ on $\left\{\xi \in \mathbb{R}^{n}:|\xi| \leq 2\right\}$ and $\operatorname{supp} \widehat{\gamma} \subseteq\{\xi \in$ $\left.\left.\mathbb{R}^{n}:|\xi|<\pi\right\}\right\}$. Define $\gamma_{\nu}(x)=2^{\nu n} \gamma\left(2^{\nu} x\right)$ for $\nu \in \mathbb{Z}$. Since $\widehat{\gamma}_{\nu}=\widehat{\gamma}\left(2^{\nu} \xi\right)$, $\operatorname{supp} \widehat{\gamma}_{\nu} \subseteq\left\{\xi \in \mathbb{R}^{n}:|\xi|<2^{\nu} \pi\right\}$.

Lemma 4.3. For $\nu \in \mathbb{Z}$ let $\vec{g} \in E_{\nu}$ and fix $x \in Q_{\nu k}$, where $k \in \mathbb{Z}^{n}$. Then for any $y \in \mathbb{R}^{n}$ and $\gamma \in \Gamma$,

$$
\vec{g}(y)=\sum_{l \in \mathbb{Z}^{n}} 2^{-\nu n} \vec{g}\left(2^{-\nu} l+x\right) \gamma_{\nu}\left(y-\left(2^{-\nu} l+x\right)\right) .
$$

Proof. Denote $\vec{g}^{x}(y)=\vec{g}(y+x)$. Trivially, $\vec{g}(y)=\vec{g}^{x}(y-x)$. Note that $\left(\vec{g}^{x}\right)^{\wedge}(\xi)=e^{i x \xi} \widehat{\vec{g}}(\xi)$, and so $\operatorname{supp}\left(\vec{g}^{x}\right)^{\wedge}=\operatorname{supp} \widehat{\vec{g}}$. Therefore, by (20) applied to $\vec{g}^{x}$,

$$
\vec{g}(y)=\vec{g}^{x}(y-x)=\sum_{l \in \mathbb{Z}^{n}} 2^{-\nu n} \vec{g}^{x}\left(2^{-\nu} l\right) \gamma_{\nu}\left(y-x-2^{-\nu} l\right),
$$

which is (21).

Lemma 4.4. If $\left\{A_{Q}\right\}$ is a doubling sequence of order $p$, then for $\vec{s}_{Q}=$ $\left\langle\vec{f}, \varphi_{Q}\right\rangle$,

$$
\left\|\left\{\vec{s}_{Q}\right\}_{Q}\right\|_{\dot{b}_{p}^{\alpha q}\left(\left\{A_{Q}\right\}\right)} \leq c\|\vec{f}\|_{\dot{B}_{p}^{\alpha q}\left(\left\{A_{Q}\right\}\right)} .
$$

Proof. Note that $\vec{s}_{Q}=|Q|^{1 / 2}\left(\widetilde{\varphi}_{\nu} * \vec{f}\right)\left(2^{-\nu} k\right)$ for $Q=Q_{\nu k}$, where $\widetilde{\varphi}(x)=$ $\overline{\varphi(-x)}$. Let $\left\|\left\{\vec{s}_{Q}\right\}_{Q}\right\|_{\dot{b}_{p}^{\alpha q}\left(\left\{A_{Q}\right\}\right)}=:\left\|\left\{J_{\nu}^{1 / p}\right\}_{\nu}\right\|_{l_{q}^{\alpha}}$, where

$$
J_{\nu}=\sum_{k \in \mathbb{Z}^{n}} \int_{Q_{\nu k}}\left\|A_{Q_{\nu k}}\left(\widetilde{\varphi}_{\nu} * \vec{f}\right)\left(2^{-\nu} k\right)\right\|^{p} d x .
$$

Since $\widetilde{\varphi}_{\nu} * \vec{f} \in E_{\nu}$, Lemma 4.3 implies

$$
\left(\widetilde{\varphi}_{\nu} * \vec{f}\right)\left(2^{-\nu} k\right)=\sum_{l \in \mathbb{Z}^{n}}\left(\widetilde{\varphi}_{\nu} * \vec{f}\right)\left(2^{-\nu} l+x\right) \gamma\left(k-l-2^{\nu} x\right), \quad x \in Q_{\nu k},
$$


for some $\gamma \in \Gamma$. Then

$$
\begin{aligned}
J_{\nu} & \leq \sum_{k \in \mathbb{Z}^{n}} \int_{Q_{\nu k}}\left(\sum_{l \in \mathbb{Z}^{n}}\left\|A_{Q_{\nu k}}\left(\widetilde{\varphi}_{\nu} * \vec{f}\right)\left(2^{-\nu} l+x\right)\right\|\left|\gamma\left(k-l-2^{\nu} x\right)\right|\right)^{p} d x \\
& \leq c \sum_{k \in \mathbb{Z}^{n}} \int_{Q_{\nu k}}\left(\sum_{l \in \mathbb{Z}^{n}} \frac{\left\|A_{Q_{\nu k}}\left(\widetilde{\varphi}_{\nu} * \vec{f}\right)\left(2^{-\nu} l+x\right)\right\|}{\left(1+\left|k-l-2^{\nu} x\right|\right)^{M}}\right)^{p} d x
\end{aligned}
$$

for some $M>\beta+n$. Using the discrete Hölder inequality and the fact that $M>n$, we bring the $p$ th power inside the sum on $l$ (for $p>1$ ). Furthermore, since $\left\{A_{Q}\right\}_{Q}$ is doubling, (1) implies

$$
\left\|A_{Q_{\nu k}} \vec{u}\right\|^{p} \leq c(1+|l|)^{\beta}\left\|A_{Q_{\nu(k+l)}} \vec{u}\right\|^{p} \quad \text { for any } \vec{u} \in \mathcal{H} .
$$

Thus,

$$
J_{\nu} \leq c \sum_{k \in \mathbb{Z}^{n}} \int_{Q_{\nu k}} \sum_{l \in \mathbb{Z}^{n}} \frac{(1+|l|)^{\beta}\left\|A_{Q_{\nu(k+l)}}\left(\widetilde{\varphi}_{\nu} * \vec{f}\right)\left(2^{-\nu} l+x\right)\right\|^{p}}{\left(1+\left|k-l-2^{\nu} x\right|\right)^{M}} d x .
$$

Changing variable $\left(t=x+2^{-\nu} l\right)$ and reindexing the sum on $l$, we get

$$
\begin{aligned}
J_{\nu} & \leq c \sum_{k \in \mathbb{Z}^{n}} \int_{Q_{\nu l}} \sum_{l \in \mathbb{Z}^{n}}(1+|k-l|)^{\beta-M}\left\|A_{Q_{\nu l}}\left(\widetilde{\varphi}_{\nu} * \vec{f}\right)(t)\right\|^{p} d t \\
& \leq c \sum_{l \in \mathbb{Z}^{n}} \int_{Q_{\nu l}}\left\|A_{Q_{\nu l}}\left(\widetilde{\varphi}_{\nu} * \vec{f}\right)(t)\right\|^{p} d t=c\left\|\widetilde{\varphi}_{\nu} * \vec{f}\right\|_{L^{p}\left(\left\{A_{Q}\right\}, \nu\right)}
\end{aligned}
$$

(the sum on $k$ converges since $M-\beta>n$ ). Thus,

$$
\left\|\left\{\vec{s}_{Q}\right\}_{Q}\right\|_{\dot{b}_{p}^{\alpha q}\left(\left\{A_{Q}\right\}\right)} \leq c\|\vec{f}\|_{\dot{B}_{p}^{\alpha q}\left(\left\{A_{Q}\right\}, \widetilde{\varphi}\right)}
$$

Now we need the independence of the space $\dot{B}_{p}^{\alpha q}\left(\left\{A_{Q}\right\}\right)$ of the choice of $\varphi$ (or $\tilde{\varphi})$. We apply the same strategy as in [5, Theorem 6.6], namely, we use the proof of Corollary 4.9 below, which will imply that the last expression is equivalent to $c\|\vec{f}\|_{\dot{B}_{p}^{\alpha q}\left(\left\{A_{Q}\right\}, \varphi\right)}$ and, thus, (22) is proved.

Corollary 4.5. If $\left\{A_{Q}\right\}$ is a doubling sequence of order $p$, then for $\vec{s}_{Q}=\left\langle\vec{f}, \varphi_{Q}\right\rangle$,

$$
\left\|\left\{\vec{s}_{Q}\right\}_{Q}\right\|_{\dot{b}_{p}^{\alpha q}\left(\left\{A_{Q}^{-1}\right\}\right)} \leq c\|\vec{f}\|_{\dot{B}_{p}^{\alpha q}\left(\left\{A_{Q}^{-1}\right\}\right)}
$$

and

$$
\left\|\left\{\vec{s}_{Q}\right\}_{Q}\right\|_{\dot{b}_{p^{\prime}}^{-\alpha q^{\prime}}\left(\left\{A_{Q}^{-1}\right\}\right)} \leq c\|\vec{f}\|_{\dot{B}_{p^{\prime}}^{-\alpha q^{\prime}}\left(\left\{A_{Q}^{-1}\right\}\right)} .
$$

Proof. For (25) repeat the previous proof with each $A_{Q}$ replaced by $A_{Q}^{-1}$ and instead of the estimate (24) use

$$
\left\|A_{Q_{\nu k}}^{-1} \vec{u}\right\|^{p} \leq c(1+|l|)^{\beta}\left\|A_{Q_{\nu(k+l)}}^{-1} \vec{u}\right\|^{p} \quad \text { for any } \vec{u} \in \mathcal{H}
$$


which follows from the doubling property (1) and duality

$$
\left\|A_{Q}^{-1} \vec{u}\right\|=\sup _{\vec{v} \neq 0} \frac{|(\vec{u}, \vec{v})|}{\left\|A_{Q} \vec{v}\right\|} .
$$

For (26) use the obvious replacements for $\alpha, p, q$ and $A_{Q}$. If $1<p<\infty$, choose $M>\beta p^{\prime} / p+n$ and replace (24) by

$$
\left\|A_{Q_{\nu k}}^{-1} \vec{u}\right\|^{p^{\prime}} \leq c(1+|l|)^{\beta p^{\prime} / p}\left\|A_{Q_{\nu(k+l)}}^{-1} \vec{u}\right\|^{p^{\prime}} \quad \text { for any } \vec{u} \in \mathcal{H},
$$

which is obtained from (27) by raising to the power $p^{\prime} / p$. If $p=1\left(p^{\prime}=\infty\right)$, then replace (23) with the $L^{\infty}$ norm:

$$
J_{\nu}=\sup _{x \in \mathbb{R}^{n}} \sum_{k \in \mathbb{Z}^{n}}\left\|A_{Q_{\nu k}}^{-1}\left(\widetilde{\varphi}_{\nu} * \vec{f}\right)\left(2^{-\nu} k\right)\right\| \chi_{Q_{\nu k}}(x)
$$

and use (27) instead of (24) to get

$$
J_{\nu} \leq c \sup _{t \in \mathbb{R}^{n}} \sum_{l \in \mathbb{Z}^{n}}\left\|A_{Q_{\nu l}}^{-1}\left(\widetilde{\varphi}_{\nu} * \vec{f}\right)(t)\right\| \chi_{Q_{\nu l}}(t)=c\left\|\widetilde{\varphi}_{\nu} * \vec{f}\right\|_{L^{\infty}\left(\left\{A_{Q}^{-1}\right\}, \nu\right)} .
$$

Recall that for each admissible $\varphi \in \mathcal{A}$ there exists $\psi \in \mathcal{A}$ (see [3, Lemma 6.9]) such that

$$
\sum_{\nu \in \mathbb{Z}} \overline{\widehat{\varphi}\left(2^{\nu} \xi\right)} \cdot \widehat{\psi}\left(2^{\nu} \xi\right)=1 \quad \text { if } \xi \neq 0
$$

A pair $(\varphi, \psi)$ with $\varphi, \psi \in \mathcal{A}$ and the property (29) is referred to as a pair of mutually admissible kernels.

Lemma 4.6. Suppose $\left\{A_{Q}\right\}_{Q}$ is a doubling sequence of order $p$. Then

$$
\|\vec{f}\|_{\dot{B}_{p}^{\alpha q}\left(\left\{A_{Q}\right\}\right)} \leq c\left\|\left\{\vec{s}_{Q}(\vec{f})\right\}_{Q}\right\|_{\dot{b}_{p}^{\alpha q}\left(\left\{A_{Q}\right\}\right)} .
$$

Proof. Using $\vec{f}=\sum_{Q} \vec{s}_{Q}(\vec{f}) \psi_{Q}$, we get

$$
\begin{aligned}
& \left\|\sum_{Q} \vec{s}_{Q}(\vec{f}) \psi_{Q}\right\|_{\dot{B}_{P}^{\alpha q}\left(\left\{A_{Q}\right\}\right)} \\
& \leq\left\|\left\{\sum_{\mu \in \mathbb{Z}}\left(\sum_{l(P)=2^{-\nu}} \int_{P}\left(\sum_{l(Q)=2^{-\mu}}\left\|A_{P} \vec{s}_{Q}\right\|\left|\left(\varphi_{\nu} * \psi_{Q}\right)(x)\right|\right)^{p} d x\right)^{1 / p}\right\}_{\nu}\right\|_{l_{q}^{\alpha}} \\
& \quad=\left\|\left\{\sum_{\mu=\nu-1}^{\nu+1}\left(\sum_{l(P)=2^{-\nu}} \int_{P}\left(\sum_{l(Q)=2^{-\mu}}\left\|A_{P} \vec{s}_{Q}\right\|\left|\left(\varphi_{\nu} * \psi_{Q}\right)(x)\right|\right)^{p} d x\right)^{1 / p}\right\}_{\nu}\right\|_{l_{q}^{\alpha}} \\
& \quad=:\left\|\left\{J_{\nu}^{1 / p}\right\}_{\nu}\right\|_{l_{q}^{\alpha}},
\end{aligned}
$$

since $\varphi_{\nu} * \psi_{Q}=0$ if $|\mu-\nu|>1$. Using the convolution estimates (16) and (17) from [5], we get (for any $M>0$ )

$$
\left|\left(\varphi_{\nu} * \psi_{Q}\right)(x)\right| \leq c_{M}|Q|^{-1 / 2}\left(1+2^{\nu}\left|x-x_{Q}\right|\right)^{-M} \quad \text { if } \mu=\nu-1, \nu, \nu+1 .
$$


If $1<p<\infty$, choose $M=M_{1}+M_{2}$ with $M_{1}>\beta / p+n / p$ and $M_{2}>n / p^{\prime} ;$ if $p=1$, let $M=M_{1}>\beta+n$. Then applying the above estimate and Hölder's inequality, we obtain

$$
J_{\nu} \leq c \sum_{\mu=\nu-1}^{\nu+1} \sum_{l(P)=2^{-\nu}} \sum_{l(Q)=2^{-\mu}}\left\|A_{P} \vec{s}_{Q}\right\|^{p}|P||Q|^{-p / 2}\left(1+2^{\nu}\left|x_{P}-x_{Q}\right|\right)^{-M_{1} p} .
$$

Shifting $A_{P}$ to $A_{Q}$ by doubling, we get

$$
J_{\nu} \leq c \sum_{\mu=\nu-1}^{\nu+1} \sum_{l(Q)=2^{-\mu}}|Q|^{-p / 2}\left\|A_{Q} \vec{s}_{Q}\right\|^{p}|Q| \sum_{l(P)=2^{-\nu}} c_{\beta}\left(1+2^{\nu}\left|x_{P}-x_{Q}\right|\right)^{-M_{1} p+\beta} .
$$

Applying [5, Lemma 5.4] (Summation Lemma) to the sum on $P$, we have

$$
\begin{aligned}
J_{\nu} & \leq c \sum_{\mu=\nu-1}^{\nu+1} \sum_{l(Q)=2^{-\mu}}|Q|^{-p / 2}\left\|A_{Q} \vec{s}_{Q}\right\|^{p}|Q| \\
& =c \sum_{\mu=\nu-1}^{\nu+1}\left\|\sum_{l(Q)=2^{-\mu}}|Q|^{-1 / 2} \vec{s}_{Q} \chi_{Q}\right\|_{L^{p}\left(\left\{A_{Q}\right\}, \mu\right)} .
\end{aligned}
$$

Combining the estimates for all $J_{\nu}$ and reindexing when necessary, we get

$$
\begin{aligned}
\|\vec{f}\|_{\dot{B}_{p}^{\alpha q}\left(\left\{A_{Q}\right\}\right)} & \leq 3 c\left\|\left\{2^{\nu \alpha}\left\|\sum_{l(Q)=2^{-\nu}}|Q|^{-1 / 2} \vec{s}_{Q} \chi_{Q}\right\|_{L^{p}\left(\left\{A_{Q}\right\}, \nu\right)}\right\}_{\nu}\right\|_{l^{q}} \\
& =c\left\|\left\{\vec{s}_{Q}\right\}\right\|_{\dot{b}_{p}^{\alpha q}\left(\left\{A_{Q}\right\}\right)} .
\end{aligned}
$$

REMARK 4.7. Theorem 1.6 is obtained by combining Lemmas 4.4 and 4.6.

Corollary 4.8. If $\left\{A_{Q}\right\}_{Q}$ is doubling (of order $p$ ), then

$$
\|\vec{f}\|_{\dot{B}_{p}^{\alpha q}\left(\left\{A_{Q}^{-1}\right\}\right)} \leq c\left\|\left\{\vec{s}_{Q}(\vec{f})\right\}_{Q}\right\|_{\dot{b}_{p}^{\alpha q}\left(\left\{A_{Q}^{-1}\right\}\right)}
$$

and

$$
\|\vec{f}\|_{\dot{B}_{p^{\prime}}^{-\alpha q^{\prime}}\left(\left\{A_{Q}^{-1}\right\}\right)} \leq c\left\|\left\{\vec{s}_{Q}(\vec{f})\right\}_{Q}\right\|_{\dot{b}_{p^{\prime}}^{-\alpha q^{\prime}}\left(\left\{A_{Q}^{-1}\right\}\right)} .
$$

Proof. For (32) use the previous proof with the following shifting of $A_{P}$ to $A_{Q}$ (similar to $\left.(27)\right)$ :

$$
\left\|A_{P}^{-1} \vec{s}_{Q}\right\|^{p} \leq c_{n, \beta, p}\left(1+2^{\nu}\left|x_{P}-x_{Q}\right|\right)^{\beta}\left\|A_{Q}^{-1} \vec{s}_{Q}\right\|^{p},
$$

where $l(P)=2^{-\nu}$ and $l(Q)=2^{-\mu}$ with $\mu=\nu-1, \nu$ or $\nu+1$; for (33) use the above proof with the indices $-\alpha, q^{\prime}, p^{\prime}$; if $1<p<\infty$, take $M>\beta p^{\prime} / p+n$ and apply (34) raised to the power $p^{\prime} / p$; if $p^{\prime}=\infty$, then

$$
J_{\nu} \leq \sup _{x \in \mathbb{R}^{n}} \sum_{\mu=\nu-1}^{\nu+1} \sum_{l(P)=2^{-\nu}} \sum_{l(Q)=2^{-\mu}}\left\|A_{P}^{-1} \vec{s}_{Q}\right\|\left|\left(\varphi_{\nu} * \psi_{Q}\right)(x)\right| \chi_{P}(x) .
$$


Using the convolution estimate (31) (with $M=M_{1}>\beta+n$ ) and (34) for shifting $A_{P}^{-1}$ to $A_{Q}^{-1}$, we get

$$
J_{\nu} \leq c \sum_{\mu=\nu-1}^{\nu+1}\left\|\sum_{l(Q)=2^{-\mu}}\right\| A_{Q}^{-1} \vec{s}_{Q}\left\|\chi_{Q}\right\|_{L^{\infty}}
$$

which gives (33).

Corollary 4.9. The spaces $\dot{B}_{p}^{\alpha q}\left(\left\{A_{Q}\right\}\right), \dot{B}_{p}^{\alpha q}\left(\left\{A_{Q}^{-1}\right\}\right)$ and $\dot{B}_{p^{\prime}}^{-\alpha q^{\prime}}\left(\left\{A_{Q}^{-1}\right\}\right)$ are independent of the choice of the admissible kernel if $\left\{A_{Q}\right\}_{Q}$ is doubling (of order $p$ ).

Proof. Repeat the proof of [5, Theorem 1.8] with $W$ replaced by $A_{Q}$ and use Lemmas 4.4 and 4.6 for the space $\dot{B}_{p}^{\alpha q}\left(\left\{A_{Q}\right\}\right)$; for the space $\dot{B}_{p}^{\alpha q}\left(\left\{A_{Q}^{-1}\right\}\right)$ apply (25) and (32), and for the space $\dot{B}_{p^{\prime}}^{-\alpha q^{\prime}}\left(\left\{A_{Q}^{-1}\right\}\right)$ use (26) and (33).

5. Properties of averaging $L^{p}$ spaces. In this section we study the connection between $L^{p}\left(\left\{A_{Q}\right\}, \nu\right)$ and $L^{p}(W)$, the dual of $L^{p}\left(\left\{A_{Q}\right\}, \nu\right)$ and several convolution estimates on $L^{p}\left(\left\{A_{Q}\right\}, \nu\right)$.

Lemma 5.1. Let $W$ be a doubling matrix weight of order $p, 1 \leq p<\infty$. Then for $\vec{f} \in E_{\nu}, \nu \in \mathbb{Z}$,

$$
\|\vec{f}\|_{L^{p}(W)} \leq c\|\vec{f}\|_{L^{p}\left(\left\{A_{Q}\right\}, \nu\right)},
$$

where $\left\{A_{Q}\right\}_{Q}$ is a sequence of reducing operators generated by $W$ and $c$ is independent of $\nu$.

Proof. Using the notation $W_{\nu}(t)=W\left(2^{-\nu} t\right)$ and $\vec{f}_{\nu}(t)=\vec{f}\left(2^{-\nu} t\right)$, we write

$$
\|\vec{f}\|_{L^{p}(W)}^{p}=\sum_{k \in \mathbb{Z}^{n}} \int_{Q_{\nu k}}\left\|W^{1 / p}(t) \vec{f}(t)\right\|^{p} d t=\sum_{k \in \mathbb{Z}^{n}} 2^{-\nu n} \int_{Q_{0 k}}\left\|W_{\nu}^{1 / p}(t) \vec{f}_{\nu}(t)\right\|^{p} d t .
$$

Since $\vec{f}_{\nu} \in E_{0}$, there exists $\gamma \in \Gamma$ such that $\vec{f}_{\nu}=\vec{f}_{\nu} * \gamma$. Using the decay of $\gamma$ and Hölder's inequality, we get

$$
\|\vec{f}\|_{L^{p}(W)}^{p} \leq \sum_{k \in \mathbb{Z}^{n}} 2^{-\nu n} \int_{Q_{0 k}} \sum_{m \in \mathbb{Z}^{n}} \int_{Q_{0 m}} \frac{\left\|W_{\nu}^{1 / p}(t) \vec{f}_{\nu}(y)\right\|^{p}}{(1+|m-k|)^{M}} d y d t
$$

for some $M>\beta+n$. Observe that $\left\|A_{Q_{\nu k}} \vec{f}_{\nu}(y)\right\|^{p} \approx \int_{Q_{0 k}}\left\|W_{\nu}^{1 / p}(t) \overrightarrow{f_{\nu}}(y)\right\|^{p} d t$. Using the doubling property of $W$ to shift $A_{Q_{\nu k}}$ to $A_{Q_{\nu m}}$ (see (24)), we obtain

$$
\begin{aligned}
\|\vec{f}\|_{L^{p}(W)}^{p} & \leq c \sum_{m \in \mathbb{Z}^{n}} \sum_{k \in \mathbb{Z}^{n}} 2^{-\nu n} \int_{Q_{0 m}}(1+|m-k|)^{-(M-\beta)}\left\|A_{Q_{\nu m}} \vec{f}_{\nu}(y)\right\|^{p} d y \\
& \leq c \sum_{m \in \mathbb{Z}^{n}} \int_{Q_{0 m}}\left\|A_{Q_{\nu m}} \vec{f}_{\nu}(y)\right\|^{p} d y
\end{aligned}
$$


where the sum on $k$ converges since $M>\beta+n$. Changing variables $x=2^{-\nu} y$, we get the desired inequality (35).

Corollary 5.2. Let $\alpha \in \mathbb{R}, 0<q \leq \infty$ and $1 \leq p<\infty$. If $W$ is doubling (of order $p$ ) and $\left\{A_{Q}\right\}_{Q}$ is a sequence of reducing operators generated by $W$, then

$$
\dot{B}_{p}^{\alpha q}\left(\left\{A_{Q}\right\}\right) \subseteq \dot{B}_{p}^{\alpha q}(W) .
$$

Proof. Since $\varphi_{\nu} * \vec{f} \in E_{\nu}$, the previous lemma implies

$$
\begin{aligned}
\|\vec{f}\|_{\dot{B}_{p}^{\alpha q}(W)} & =\left\|\left\{2^{\nu \alpha}\left\|\varphi_{\nu} * \vec{f}\right\|_{L^{p}(W)}\right\}_{\nu}\right\|_{l^{q}} \\
& \leq c\left\|\left\{2^{\nu \alpha}\left\|\varphi_{\nu} * \vec{f}\right\|_{L^{p}\left(\left\{A_{Q}\right\}, \nu\right)}\right\}_{\nu}\right\|_{l^{q}}=c\|\vec{f}\|_{\dot{B}_{p}^{\alpha q}\left(\left\{A_{Q}\right\}\right)} .
\end{aligned}
$$

Lemma 5.3. Let $1 \leq p<\infty$ and $W$ satisfy any of (A1)-(A3). Suppose $\vec{f} \in E_{\nu}, \nu \in \mathbb{Z}$. Then

$$
\|\vec{f}\|_{L^{p}\left(\left\{A_{Q}\right\}, \nu\right)} \leq c\|\vec{f}\|_{L^{p}(W)},
$$

where $\left\{A_{Q}\right\}_{Q}$ is a sequence of reducing operators produced by $W$ and $c$ is independent of $\nu$.

Proof. Using the definition of reducing operators, we write

$$
\begin{aligned}
\|\vec{f}\|_{L^{p}\left(\left\{A_{Q}\right\}, \nu\right)} & \approx \sum_{k \in \mathbb{Z}^{n}} \int_{Q_{\nu k}} \frac{1}{\left|Q_{\nu k}\right|} \int_{Q_{\nu k}}\left\|W^{1 / p}(t) \vec{f}(x)\right\|^{p} d t d x \\
& =\sum_{k \in \mathbb{Z}^{n}} \int_{Q_{0 k}} \int_{Q_{\nu k}}\left\|W^{1 / p}(t) \vec{f}_{\nu}(y)\right\|^{p} d t d y,
\end{aligned}
$$

by changing variables $x=2^{-\nu} y$ and denoting $\vec{f}_{\nu}(y)=\vec{f}\left(2^{-\nu} y\right)$. Note that $\overrightarrow{f_{\nu}} \in E_{0}$. Applying the decomposition of an exponential type function (Lemma 4.2) to $\overrightarrow{f_{\nu}}=\overrightarrow{f_{\nu}} * \gamma$ for $\gamma \in \Gamma$ and Hölder's inequality (choose $M>\beta+n$ ), the last expression is bounded by

$$
\begin{aligned}
& c \sum_{k \in \mathbb{Z}^{n}} \int_{Q_{0 k}} \int_{Q_{\nu k}} \sum_{m \in \mathbb{Z}^{n}} \frac{\left\|W^{1 / p}(t) \vec{f}_{\nu}(m)\right\|^{p}}{(1+|y-m|)^{M}} d t d y \\
& \quad \leq c \sum_{m \in \mathbb{Z}^{n}} \sum_{k \in \mathbb{Z}^{n}} \frac{1}{(1+|k-m|)^{M-\beta}} \int_{Q_{0 k}} \int_{Q_{\nu m}}\left\|W^{1 / p}(t) \vec{f}_{\nu}(m)\right\|^{p} d t d y,
\end{aligned}
$$

by applying the doubling property of $W$ (any of (A1)-(A3) implies that $W$ is doubling). Integrating on $y$ and summing on $k(M>\beta+n)$, we bound the previous line by

$$
c \sum_{m \in \mathbb{Z}^{n}} \int_{Q_{\nu m}}\left\|W^{1 / p}(t) \vec{f}_{\nu}(m)\right\|^{p} d t=c 2^{-\nu n} \sum_{m \in \mathbb{Z}^{n}} \int_{Q_{0 m}}\left\|W_{\nu}^{1 / p}(t) \vec{f}_{\nu}(m)\right\|^{p} d t,
$$


again by changing variables. Now applying [5, Lemmas 6.3 and 6.5] (this is where (A1)-(A3) come into play), we bound the above by $c 2^{-\nu n}\left\|\vec{f}_{\nu}\right\|_{L^{p}\left(W_{\nu}\right)}^{p}$ $=c\|\vec{f}\|_{L^{p}(W)}^{p}$, which gives $(36)$.

Corollary 5.4. Let $\alpha \in \mathbb{R}, 0<q \leq \infty$ and $1 \leq p<\infty$. If $W$ satisfies any of (A1)-(A3) and $\left\{A_{Q}\right\}_{Q}$ is a sequence of reducing operators generated by $W$, then

$$
\dot{B}_{p}^{\alpha q}(W) \subseteq \dot{B}_{p}^{\alpha q}\left(\left\{A_{Q}\right\}\right) .
$$

Proof. As in the proof of Corollary 5.2, use the fact that $\varphi_{\nu} * \vec{f} \in E_{\nu}$ and Lemma 5.3.

Remark 5.5. Combining Corollaries 5.2 and 5.4, we have Lemma 1.7.

In order to establish the dual of $L^{p}\left(\left\{A_{Q}\right\}, \nu\right), 1<p<\infty$, we consider the following idea:

$$
\begin{aligned}
\|\vec{f}\|_{L^{p}\left(\left\{A_{Q}\right\}, \nu\right)}^{p} & =\sum_{Q \in Q_{\nu}} \int_{Q}\left\|A_{Q} \vec{f}(x)\right\|_{\mathcal{H}}^{p} d x \\
& =\int_{\mathbb{R}^{n}}\left(\sum_{Q \in Q_{\nu}}\left\|A_{Q} \vec{f}(x)\right\|_{\mathcal{H} \chi Q}(x)\right)^{p} d x \\
& =\int_{\mathbb{R}^{n}}\left\|\sum_{Q \in Q_{\nu}} A_{Q} \chi_{Q}(x) \vec{f}(x)\right\|_{\mathcal{H}}^{p} d x \\
& =: \int_{\mathbb{R}^{n}}\left\|U_{\nu}^{1 / p}(x) \vec{f}(x)\right\|_{\mathcal{H}}^{p} d x=\|\vec{f}\|_{L^{p}\left(U_{\nu}\right)}^{p},
\end{aligned}
$$

i.e., $L^{p}\left(\left\{A_{Q}\right\}, \nu\right)=L^{p}\left(U_{\nu}\right)$, where $U_{\nu}(x)=\sum_{Q \in Q_{\nu}} A_{Q}^{p} \chi_{Q}(x)$ is a matrix weight. Since the dual $\left[L^{p}\left(U_{\nu}\right)\right]^{*}$ can be identified with $L^{p^{\prime}}\left(U_{\nu}^{*}\right)$ with $U_{\nu}^{1 / p}(x)=\left(U_{\nu}^{*}\right)^{-1 / p^{\prime}}(x)$ (e.g. see [4] or [10]), i.e., $U_{\nu}^{*}(x)=\sum_{Q \in Q_{\nu}} A_{Q}^{-p^{\prime}} \chi_{Q}(x)$, we obtain

$$
\begin{aligned}
\|\vec{f}\|_{L^{p^{\prime}\left(U_{\nu}^{*}\right)}}^{p^{\prime}} & =\int_{\mathbb{R}^{n}}\left\|\sum_{Q \in Q_{\nu}} A_{Q}^{-1} \chi_{Q}(x) \vec{f}(x)\right\|_{\mathcal{H}}^{p^{\prime}} d x \\
& =\sum_{Q \in Q_{\nu}} \int_{Q}\left\|A_{Q}^{-1} \vec{f}(x)\right\|_{\mathcal{H}}^{p^{\prime}} d x=\|\vec{f}\|_{L^{p^{\prime}}\left(\left\{A_{Q}^{-1}\right\}, \nu\right)}^{p^{\prime}}
\end{aligned}
$$

or

$$
\left[L^{p}\left(\left\{A_{Q}\right\}, \nu\right)\right]^{*} \approx L^{p^{\prime}}\left(\left\{A_{Q}^{-1}\right\}, \nu\right) .
$$

If $p=1$, then the standard duality argument gives

$$
\left[L^{1}\left(\left\{A_{Q}\right\}, \nu\right)\right]^{*} \approx L^{\infty}\left(\left\{A_{Q}^{-1}\right\}, \nu\right) .
$$

The details are left to the reader. 
The boundedness of the convolution operator with a decaying kernel on $L^{p}\left(\left\{A_{Q}\right\}, \nu\right)$ will be helpful in the next section. We establish it here.

Lemma 5.6. Let $|\Phi(t)| \leq c /(1+|t|)^{M}$ for some $M>\beta / p+n$ and for $\nu \in \mathbb{Z}$ define $\Phi_{\nu}(t)=2^{\nu n} \Phi\left(2^{\nu} t\right)$. Let $\left\{A_{Q}\right\}_{Q}$ be a doubling matrix sequence of order $p, 1 \leq p<\infty$. Fix $\lambda, \mu, \nu \in \mathbb{Z}$. Then

(i) $\left\|\Phi_{\mu} * \vec{f}\right\|_{L^{p}\left(\left\{A_{Q}\right\}, \lambda\right)} \leq c_{0}\left(c_{1}\right)^{\lambda-\nu}\left(c_{2}\right)^{\mu-\nu}\|\vec{f}\|_{L^{p}\left(\left\{A_{Q}\right\}, \nu\right)}$,

(ii) $\left\|\Phi_{\mu} * \vec{f}\right\|_{L^{p}\left(\left\{A_{Q}^{-1}\right\}, \lambda\right)} \leq c_{0}\left(c_{3}\right)^{\lambda-\nu}\left(c_{2}\right)^{\mu-\nu}\|\vec{f}\|_{L^{p}\left(\left\{A_{Q}^{-1}\right\}, \nu\right)}$,

where $c_{1}=2^{n / p} \chi_{\{\lambda>\nu\}}+2^{(n-\beta) / p} \chi_{\{\lambda \leq \nu\}}, c_{2}=2^{n} \chi_{\{\mu>\nu\}}+2^{n-M} \chi_{\{\mu \leq \nu\}}$, $c_{3}=2^{(\beta-n) / p} \chi_{\{\lambda>\nu\}}+2^{-n / p} \chi_{\{\lambda \leq \nu\}}$, and $c_{0}$ is independent of $\lambda, \mu$ and $\nu$.

Proof. Using the decay of $\Phi$, namely,

$$
\left|\Phi_{\mu}(x-y)\right| \leq c k_{2} 2^{\nu n} /\left(1+2^{\nu}|x-y|\right)^{M},
$$

where $k_{2}=2^{(\mu-\nu) n} \chi_{\{\mu>\nu\}}+2^{(\nu-\mu)(M-n)} \chi_{\{\mu \leq \nu\}}$, we have

$$
\begin{aligned}
\left\|\Phi_{\mu} * \vec{f}\right\|_{L^{p}\left(\left\{A_{Q}\right\}, \lambda\right)}^{p} & =\sum_{Q \in Q_{\lambda}} \int_{Q}\left\|A_{Q}\left(\Phi_{\mu} * \vec{f}\right)(x)\right\|^{p} d x \\
& \leq \sum_{Q \in Q_{\lambda}} \int_{Q}\left(\int_{\mathbb{R}^{n}}\left\|A_{Q} \vec{f}(y)\right\|\left|\Phi_{\mu}(x-y)\right| d y\right)^{p} d x \\
& \leq c \sum_{Q \in Q_{\lambda}} \int_{Q}\left(\int_{\mathbb{R}^{n}} \frac{k_{2} 2^{\nu n}\left\|A_{Q} \vec{f}(y)\right\|}{\left(1+2^{\nu}|x-y|\right)^{M}} d y\right)^{p} d x \\
& \approx c \sum_{k \in \mathbb{Z}^{n}} \int_{Q_{\lambda k}}\left(\sum_{m \in \mathbb{Z}^{n}} \int_{Q_{\nu m}} \frac{k_{2} 2^{\nu n}\left\|A_{Q_{\lambda k}} \vec{f}(y)\right\|}{\left(1+2^{\nu}\left|x-x_{Q_{\nu m}}\right|\right)^{M}} d y\right)^{p} d x .
\end{aligned}
$$

Since $\left\{A_{Q}\right\}_{Q}$ is doubling, we "shift" $A_{Q_{\lambda k}}$ to $A_{Q_{\nu m}}$ :

$$
\left\|A_{Q_{\lambda k}} \vec{f}(y)\right\| \leq c k_{1}\left(1+2^{\nu}\left|x-x_{Q_{\nu m}}\right|\right)^{\beta / p}\left\|A_{Q_{\nu m}} \vec{f}(y)\right\| \quad \text { for } x \in Q_{\lambda k},
$$

where $k_{1}=2^{(\lambda-\nu) n / p} \chi_{\{\lambda>\nu\}}+2^{(\nu-\lambda)(\beta-n) / p} \chi_{\{\lambda \leq \nu\}}$. Substituting (38) into the convolution estimate, we get

$$
\left\|\Phi_{\mu} * \vec{f}\right\|_{L^{p}\left(\left\{A_{Q}\right\}, \lambda\right)}^{p} \leq c \int_{\mathbb{R}^{n}}\left(\sum_{m \in \mathbb{Z}^{n}} \int_{Q_{\nu m}} \frac{k_{1} k_{2} 2^{\nu n}\left\|A_{Q_{\nu m}} \vec{f}(y)\right\|}{\left(1+\left|2^{\nu} x-m\right|\right)^{M-\beta / p}} d y\right)^{p} d x .
$$

Using the discrete Hölder inequality on the sum inside and then Jensen's inequality to bring $p$ th power inside the integral (if $p>1$ ), the last line is bounded above by 


$$
\begin{aligned}
c k_{1}^{p} k_{2}^{p} \int_{\mathbb{R}^{n}} & \left(\sum_{l \in \mathbb{Z}^{n}} \frac{1}{\left(1+\left|2^{\nu} x-l\right|\right)^{M-\beta / p}}\right)^{p / p^{\prime}} \\
& \times\left(\sum_{m \in \mathbb{Z}^{n}} \int_{Q_{\nu m}} \frac{2^{\nu n}\left\|A_{Q_{\nu m}} \vec{f}(y)\right\|^{p}}{\left(1+\left|2^{\nu} x-m\right|\right)^{M-\beta / p}} d y\right) d x \\
\leq & c k_{1}^{p} k_{2}^{p} \sum_{m \in \mathbb{Z}^{n}} \int_{\mathbb{R}^{n}} \frac{2^{\nu n}}{\left(1+\left|2^{\nu} x-m\right|\right)^{M-\beta / p}} \int_{Q_{\nu m}}\left\|A_{Q_{\nu m}} \vec{f}(y)\right\|^{p} d y d x
\end{aligned}
$$

since $M-\beta / p>n$, the sum on $l$ converges (independently of $x$ ). Changing variables $\left(t=2^{\nu} x\right)$ and observing that the integral on $t$ converges (independently of $m$ ), again since $M-\beta / p>n$, we obtain

$$
\left\|\Phi_{\mu} * \vec{f}\right\|_{L^{p}\left(\left\{A_{Q}\right\}, \mu\right)}^{p} \leq c k_{1}^{p} k_{2}^{p} \sum_{m \in \mathbb{Z}^{n}} \int_{Q_{\nu m}}\left\|A_{Q_{\nu m}} \vec{f}(y)\right\|^{p} d y .
$$

Put $c_{1}=k_{1}^{1 /(\lambda-\nu)}$ and $c_{2}=k_{2}^{1 /(\mu-\nu)}$. Then part (i) is proved.

For the second part observe that (1) ("shift" $A_{Q_{\nu m}}$ to $A_{Q_{\lambda k}}$ ) together with

$$
\left\|A_{Q}^{-1} \vec{v}\right\|=\sup _{\vec{u} \neq 0} \frac{|(\vec{v}, \vec{u})|}{\left\|A_{Q} \vec{u}\right\|}
$$

implies

$$
\left\|A_{Q_{\lambda k}}^{-1} \vec{f}(y)\right\| \leq c k_{3}\left(1+2^{\nu}\left|x-x_{Q_{\nu m}}\right|\right)^{\beta / p}\left\|A_{Q_{\nu m}}^{-1} \vec{f}(y)\right\|, \quad x \in Q_{\lambda k},
$$

where $k_{3}=2^{(\lambda-\nu)(\beta-n) / p} \chi_{\{\lambda>\nu\}}+2^{(\nu-\lambda) n / p} \chi_{\{\lambda \leq \nu\}}$. Note that (39) is similar to (38), so previous estimates with each $A_{Q}$ replaced by $A_{Q}^{-1}$ prove (ii) with $c_{3}=k_{3}^{1 /(\lambda-\nu)}$.

REMARK 5.7. Recall that $\left\|A_{Q}^{-1} \vec{u}\right\| \leq c\left\|A_{Q}^{\#} \vec{u}\right\|$ for any $\vec{u} \in \mathcal{H}$ (since $\left.\left\|\left(A_{Q}^{\#} A_{Q}\right)^{-1}\right\| \leq c\right)$. Suppose that $W^{-p^{\prime} / p}$ is a doubling matrix of order $p^{\prime}$, $1<p^{\prime}<\infty$, with the doubling exponent $\beta^{*}$ (instead of the assumption that $W$ is doubling of order $p$ ). Then

$$
\left\|A_{Q_{\lambda k}}^{-1} \vec{f}(y)\right\| \leq c\left\|A_{Q_{\lambda k}}^{\#} \vec{f}(y)\right\| \leq c k_{1}^{*}\left(1+2^{\nu}\left|x-x_{Q_{\nu m}}\right|\right)^{\beta^{*} / p^{\prime}}\left\|A_{Q_{\nu m}}^{\#} \vec{f}(y)\right\|,
$$

(where $k_{1}^{*}=2^{(\lambda-\nu) n / p^{\prime}} \chi_{\{\lambda>\nu\}}+2^{(\nu-\lambda)\left(\beta^{*}-n\right) / p^{\prime}} \chi_{\{\lambda \leq \nu\}}$, i.e., $k_{1}$ with $\beta$ replaced by $\beta^{*}$ and $p$ by $p^{\prime}$ ) holds instead of (38). Choosing $M>\beta^{*} / p^{\prime}+n$ in the previous lemma, we get

(iii) $\left\|\Phi_{\mu} * \vec{f}\right\|_{L^{p}\left(\left\{A_{Q}^{-1}\right\}, \lambda\right)} \leq c_{0}\left(c_{1}^{*}\right)^{\lambda-\nu}\left(c_{2}\right)^{\mu-\nu}\|\vec{f}\|_{L^{p}\left(\left\{A_{Q}^{\#}\right\}, \nu\right)}, 1<p<\infty$.

REMARK 5.8. A similar convolution estimate can be proved for $L^{p}(W)$ spaces, $1<p<\infty$ :

$$
\|\Phi * \vec{f}\|_{L^{p}(W)} \leq c\|\vec{f}\|_{L^{p}(W)} .
$$


Recall that if $\Phi$ were to be a Calderón-Zygmund singular kernel $K$, then $\|K * \vec{f}\|_{L^{p}(W)} \leq c\|\vec{f}\|_{L^{p}(W)}$ if $W \in A_{p}$ (see [4], [8], [10]). Conversely, if (40) holds for every $\Phi \in \mathcal{S}$, then $W \in A_{p}$ is necessary (see [6]).

6. Duality of continuous Besov spaces. Now we shift our attention to continuous Besov spaces and our task is to construct $\left[\dot{B}_{p}^{\alpha q}\left(\left\{A_{Q}\right\}\right)\right]^{*}$ and eventually $\left[\dot{B}_{p}^{\alpha q}(W)\right]^{*}$.

Lemma 6.1. Let $\left\{A_{Q}\right\}_{Q}$ be a doubling matrix sequence of order $p, 1 \leq$ $p<\infty$. Let $\alpha \in \mathbb{R}$ and $0<q<\infty$. Then

$$
\dot{B}_{p^{\prime}}^{-\alpha q^{\prime}}\left(\left\{A_{Q}^{-1}\right\}\right) \subseteq\left[\dot{B}_{p}^{\alpha q}\left(\left\{A_{Q}\right\}\right)\right]^{*} .
$$

Proof. Take $\varphi, \psi \in \mathcal{A}$ with the mutual property (29). Let $\widetilde{\psi}(x)=\overline{\psi(-x)}$. Note that $\widehat{\widetilde{\psi}}(\xi)=\overline{\widehat{\psi}}(\xi)$. Let $\vec{f} \in \dot{B}_{p}^{\alpha q}\left(\left\{A_{Q}\right\}\right)$ and $\vec{g} \in \dot{B}_{p^{\prime}}^{-\alpha q^{\prime}}\left(\left\{A_{Q}^{-1}\right\}\right)$. First consider $\mathcal{S}_{0}=\{f \in \mathcal{S}: 0 \notin \operatorname{supp} \widehat{f}\}$ a dense subspace of $\dot{B}_{p}^{\alpha q}\left(\left\{A_{Q}\right\}\right)$ (see [6]) and take $\vec{f}$ with $(\vec{f})_{i} \in \mathcal{S}_{0}, i=1, \ldots, m$ (and $\vec{g}$ with $\left.(\vec{g})_{i} \in \mathcal{S}^{\prime}\right)$. Then

$$
\vec{g}=\sum_{\nu \in \mathbb{Z}} \vec{g} *\left(\varphi_{\nu} * \widetilde{\psi}_{\nu}\right) \quad \text { since } \quad \sum_{\nu \in \mathbb{Z}}\left(\varphi_{\nu} * \widetilde{\psi}_{\nu}\right)^{\wedge}(\xi)=1 \quad \text { by }(29),
$$

and

$$
\begin{aligned}
\vec{g}(\vec{f}) & =\sum_{\nu \in \mathbb{Z}}\left(\vec{g} *\left(\varphi_{\nu} * \widetilde{\psi}_{\nu}\right), \vec{f}\right)=\sum_{\nu \in \mathbb{Z}}\left(\left(\vec{g} * \varphi_{\nu}\right),\left(\vec{f} * \psi_{\nu}\right)\right) \\
& =\sum_{\nu \in \mathbb{Z}} \sum_{Q \in Q_{\nu}} \int_{Q}\left\langle A_{Q} A_{Q}^{-1}\left(\vec{g} * \varphi_{\nu}\right)(x),\left(\vec{f} * \psi_{\nu}\right)(x)\right\rangle_{\mathcal{H}} d x \\
& \leq \sum_{\nu \in \mathbb{Z}} \sum_{Q \in Q_{\nu}} \int_{\mathbb{R}^{n}}\left\|A_{Q}^{-1}\left(\vec{g} * \varphi_{\nu}\right)(x)\right\|_{\mathcal{H}}\left\|A_{Q}\left(\vec{f} * \psi_{\nu}\right)(x)\right\|_{\mathcal{H}} \chi_{Q}(x) d x
\end{aligned}
$$

by the self-adjointness of each $A_{Q}$ and the Cauchy-Schwarz inequality. Using Hölder's inequality several times, we obtain

$$
\begin{aligned}
|\vec{g}(\vec{f})| \leq & \sum_{\nu \in \mathbb{Z}} 2^{\nu \alpha}\left\|\left(\vec{f} * \psi_{\nu}\right)\right\|_{L^{p}\left(\left\{A_{Q}\right\}, \nu\right)} \cdot 2^{-\nu \alpha}\left\|\left(\vec{g} * \varphi_{\nu}\right)\right\|_{L^{p^{\prime}}\left(\left\{A_{Q}^{-1}\right\}, \nu\right)} \\
\leq & \left\|\left\{2^{\nu \alpha}\left\|\left(\vec{f} * \psi_{\nu}\right)\right\|_{L^{p}\left(\left\{A_{Q}\right\}, \nu\right)}\right\}_{\nu}\right\|_{l^{q}} \\
& \times\left\|\left\{2^{-\nu \alpha}\left\|\left(\vec{g} * \varphi_{\nu}\right)\right\|_{L^{p^{\prime}}\left(\left\{A_{Q}^{-1}\right\}, \nu\right)}\right\}_{\nu}\right\|_{l^{\prime}}
\end{aligned}
$$

if $1<q<\infty$, and if $0<q \leq 1$, we bound (42) by

$$
\begin{aligned}
& \left\|\left\{2^{\nu \alpha}\left\|\left(\vec{f} * \psi_{\nu}\right)\right\|_{L^{p}\left(\left\{A_{Q}\right\}, \nu\right)}\right\}_{\nu}\right\|_{l^{1}}\left\|\left\{2^{-\nu \alpha}\left\|\left(\vec{g} * \varphi_{\nu}\right)\right\|_{L^{p^{\prime}}\left(\left\{A_{Q}^{-1}\right\}, \nu\right)}\right\}_{\nu}\right\|_{l^{\infty}} \\
& \quad \leq\left\|\left\{2^{\nu \alpha}\left\|\left(\vec{f} * \psi_{\nu}\right)\right\|_{L^{p}\left(\left\{A_{Q}\right\}, \nu\right)}\right\}_{\nu}\right\|_{l^{q}}\left\|\left\{2^{-\nu \alpha}\left\|\left(\vec{g} * \varphi_{\nu}\right)\right\|_{L^{p^{\prime}}\left(\left\{A_{Q}^{-1}\right\}, \nu\right)}\right\}_{\nu}\right\|_{l^{\infty}} .
\end{aligned}
$$


Combining cases and using the fact that $\dot{B}_{p}^{\alpha q}\left(\left\{A_{Q}\right\}\right)$ and $\dot{B}_{p^{\prime}}^{-\alpha q^{\prime}}\left(\left\{A_{Q}^{-1}\right\}\right)$ are independent of the choice of the admissible kernel if $\left\{A_{Q}\right\}_{Q}$ is doubling (Corollary 4.9), we get

$$
|\vec{g}(\vec{f})| \leq\|\vec{f}\|_{\dot{B}_{p}^{\alpha q}\left(\left\{A_{Q}\right\}\right)}\|\vec{g}\|_{\dot{B}_{p^{\prime}}^{-\alpha q^{\prime}}\left(\left\{A_{Q}^{-1}\right\}\right)} .
$$

Since $\mathcal{S}_{0}$ is dense in $\dot{B}_{p}^{\alpha q}\left(\left\{A_{Q}\right\}\right)$, we get the above inequality for any $\vec{f} \in$ $\dot{B}_{p}^{\alpha q}\left(\left\{A_{Q}\right\}\right)$. Thus, $\vec{g} \in \dot{B}_{p^{\prime}}^{-\alpha q^{\prime}}\left(\left\{A_{Q}^{-1}\right\}\right)$ belongs to $\left[\dot{B}_{p}^{\alpha q}\left(\left\{A_{Q}\right\}\right)\right]^{*}$ and $\|\vec{g}\|_{\text {oper }}$ $\leq\|\vec{g}\|_{\dot{B}_{p^{\prime}}^{-\alpha q^{\prime}}\left(\left\{A_{Q}^{-1}\right\}\right)}$.

Lemma 6.2. Let $\alpha \in \mathbb{R}, 1 \leq p<\infty, 0<q<\infty$ and $\left\{A_{Q}\right\}_{Q}$ be a doubling sequence of order $p$. Then

$$
\left[\dot{B}_{p}^{\alpha q}\left(\left\{A_{Q}\right\}\right)\right]^{*} \subseteq \dot{B}_{p^{\prime}}^{-\alpha q^{\prime}}\left(\left\{A_{Q}^{-1}\right\}\right) .
$$

Proof. Let $l \in\left[\dot{B}_{p}^{\alpha q}\left(\left\{A_{Q}\right\}\right)\right]^{*}$. We show that there exists $\vec{g} \in \dot{B}_{p^{\prime}}^{-\alpha q^{\prime}}\left(\left\{A_{Q}^{-1}\right\}\right)$ such that $l(\vec{f})=\vec{g}(\vec{f})=(\vec{f}, \vec{g})$ for any $\vec{f} \in \dot{B}_{p}^{\alpha q}\left(\left\{A_{Q}\right\}\right)$.

CASE $1 \leq q<\infty$. Take $\vec{f} \in \dot{B}_{p}^{\alpha q}\left(\left\{A_{Q}\right\}\right)$, and for any $\nu \in \mathbb{Z}$ write $\overrightarrow{f_{\nu}}=\vec{f} * \varphi_{\nu}$. Set $T$ by $T\left(\left\{\vec{f}_{\nu}\right\}_{\nu}\right)=l(\vec{f})$, so $T$ is defined on a subspace of $l_{q}^{\alpha}\left(L^{p}\left(\left\{A_{Q}\right\}, \nu\right)\right)$. Since $l$ is bounded, so is $T$ :

$$
\left|T\left(\left\{\vec{f}_{\nu}\right\}_{\nu}\right)\right|=|l(\vec{f})| \leq c\|\vec{f}\|_{\dot{B}_{p}^{\alpha q}\left(\left\{A_{Q}\right\}\right)}=c\left\|\left\{\vec{f}_{\nu}\right\}_{\nu}\right\|_{l_{q}^{\alpha}\left(L^{p}\left(\left\{A_{Q}\right\}, \nu\right)\right)} .
$$

Extend $T$, denote by $\widetilde{T}$ the extension onto all of $l_{q}^{\alpha}\left(L^{p}\left(\left\{A_{Q}\right\}, \nu\right)\right.$ ) (note: $q \geq 1)$. Since $\left[l^{q}(X)\right]^{*} \approx l^{q^{\prime}}\left(X^{*}\right)$ (cf. Section 3 or $[1$, Chapter 8]), we have $\left[l_{q}^{\alpha}\left(L^{p}\left(\left\{A_{Q}\right\}, \nu\right)\right)\right]^{*} \approx l_{q^{\prime}}^{-\alpha}\left(\left[L^{p}\left(\left\{A_{Q}\right\}, \nu\right)\right]^{*}\right) \approx l_{q^{\prime}}^{-\alpha}\left(L^{p^{\prime}}\left(\left\{A_{Q}^{-1}\right\}, \nu\right)\right)$ by $(37)$. Thus, there exists a vector-valued sequence $\left\{\vec{g}_{\nu}\right\}_{\nu \in \mathbb{Z}} \in l_{q^{\prime}}^{-\alpha}\left(L^{p^{\prime}}\left(\left\{A_{Q}^{-1}\right\}, \nu\right)\right)$ such that $\left\|\left\{\vec{g}_{\nu}\right\}_{\nu \in \mathbb{Z}}\right\|_{l_{q^{\prime}}^{-\alpha}\left(L^{p^{\prime}}\left(\left\{A_{Q}^{-1}\right\}, \nu\right)\right)} \leq\|l\|$ and, for any $\vec{f} \in \dot{B}_{p}^{\alpha q}\left(\left\{A_{Q}\right\}\right)$,

$$
\begin{aligned}
l(\vec{f}) & =\widetilde{T}\left(\left\{\vec{f}_{\nu}\right\}\right)=T\left(\left\{\vec{f}_{\nu}\right\}\right)=\left\{\vec{g}_{\nu}\right\}\left(\left\{\vec{f}_{\nu}\right\}\right) \\
& =\sum_{\nu \in \mathbb{Z}} \int_{\mathbb{R}^{n}}\left\langle\vec{f}_{\nu}(x), \vec{g}_{\nu}(x)\right\rangle_{\mathcal{H}} d x \\
& =\sum_{\nu \in \mathbb{Z}} \int_{\mathbb{R}^{n}}\left\langle\left(f * \varphi_{\nu}\right)(x), \vec{g}_{\nu}(x)\right\rangle_{\mathcal{H}} d x \\
& =\sum_{\nu \in \mathbb{Z}} \int_{\mathbb{R}^{n}}\left\langle\vec{f}(x),\left(\vec{g}_{\nu} * \widetilde{\varphi}_{\nu}\right)(x)\right\rangle_{\mathcal{H}} d x .
\end{aligned}
$$

Define $\vec{g}(x)=\sum_{\nu \in \mathbb{Z}}\left(\vec{g}_{\nu} * \widetilde{\varphi}_{\nu}\right)(x)$. Then $l(\vec{f})=(\vec{f}, \vec{g})$. Moreover, for any $\psi \in \mathcal{A}$ (by Corollary 4.9), 


$$
\begin{aligned}
\|\vec{g}\|_{\dot{B}_{p^{\prime}}^{-\alpha q^{\prime}}\left(\left\{A_{Q}^{-1}\right\}\right)} & \approx\left\|\left\{\left\|\sum_{\nu \in \mathbb{Z}} \vec{g}_{\nu} * \widetilde{\varphi}_{\nu} * \psi_{\mu}\right\|_{L^{p^{\prime}}\left(\left\{A_{Q}^{-1}\right\}, \mu\right)}\right\}_{\mu}\right\|_{l_{q^{\prime}}^{-\alpha}} \\
& \leq\left\|\left\{\sum_{\nu \in \mathbb{Z}}\left\|\vec{g}_{\nu} * \widetilde{\varphi}_{\nu} * \psi_{\mu}\right\|_{L^{p^{\prime}}\left(\left\{A_{Q}^{-1}\right\}, \mu\right)}\right\}_{\mu}\right\|_{l_{q^{\prime}}^{-\alpha}} \\
& =\left\|\left\{\sum_{\nu=\mu-1}^{\mu+1}\left\|\vec{g}_{\nu} * \widetilde{\varphi}_{\nu} * \psi_{\mu}\right\|_{L^{p^{\prime}}\left(\left\{A_{Q}^{-1}\right\}, \mu\right)}\right\}_{\mu}\right\|_{l_{q^{\prime}}^{-\alpha}},
\end{aligned}
$$

since $\operatorname{supp} \widehat{\psi}_{\mu} \subseteq\left\{\xi: 2^{\mu-1} \leq|\xi| \leq 2^{\mu+1}\right\}$ and so $\widetilde{\varphi}_{\nu} * \psi_{\mu}=0$ if $|\mu-\nu|>1$. Reindexing the inner sum, we get

$$
\|\vec{g}\|_{\dot{B}_{p^{\prime}}^{-\alpha q^{\prime}}\left(\left\{A_{Q}^{-1}\right\}\right)}^{q^{\prime}} \leq c \sum_{\mu \in \mathbb{Z}} 2^{-\mu \alpha q^{\prime}} \sum_{j=-1}^{1}\left\|\vec{g}_{\mu+j} * \widetilde{\varphi}_{\mu} * \psi_{\mu+j}\right\|_{L^{p^{\prime}}\left(\left\{A_{Q}^{-1}\right\}, \mu\right)}^{q^{\prime}} .
$$

Since $\left\{A_{Q}\right\}_{Q}$ is doubling and the sum on $j$ is finite, we apply Lemma 5.6(ii) to get

$$
\|\vec{g}\|_{\dot{B}_{p^{\prime}}^{-\alpha q^{\prime}}\left(\left\{A_{Q}^{-1}\right\}\right)} \leq c^{\prime}\left\|\left\{2^{-\mu \alpha}\left\|\vec{g}_{\mu}\right\|_{L^{p^{\prime}}\left(\left\{A_{Q}^{-1}\right\}, \mu\right)}\right\}_{\mu}\right\|_{l^{q^{\prime}}} \leq\|l\|
$$

CASE $0<q<1$. Take $\vec{f}$ with $\overrightarrow{f_{i}} \in \mathcal{S}_{0}\left(0 \notin \operatorname{supp}\left(\overrightarrow{f_{i}}\right)^{\wedge}\right)$. Since $\varphi \in \mathcal{S}_{0}$, for $\nu \in \mathbb{Z}$ by definition of convolution and then boundedness of $l$, we have

$$
\left|\left(l * \varphi_{\nu}\right)(\vec{f})\right|=\left|l\left(\vec{f} * \widetilde{\varphi}_{\nu}\right)\right| \leq\|l\|\left\|\vec{f} * \widetilde{\varphi}_{\nu}\right\|_{\dot{B}_{p}^{\alpha q}\left(\left\{A_{Q}\right\}\right)} .
$$

Note that each component of $l * \varphi_{\nu}$ is a $\mathcal{C}^{\infty}$-function and also

$$
\left\|\vec{f} * \widetilde{\varphi}_{\nu}\right\|_{\dot{B}_{p}^{\alpha q}\left(\left\{A_{Q}\right\}\right)} \leq 2^{\nu \alpha} \sum_{\mu=\nu-1}^{\nu+1}\left\|\vec{f} * \widetilde{\varphi}_{\nu}\right\|_{L^{p}\left(\left\{A_{Q}\right\}, \mu\right)} \leq c 2^{\nu \alpha}\|\vec{f}\|_{L^{p}\left(\left\{A_{Q}\right\}, \nu\right)}
$$

by Lemma $5.6(\mathrm{i})$. Substituting this estimate into $(44)$, we get $\left|\left(l * \varphi_{\nu}\right)(\vec{f})\right| \leq$ $c 2^{\nu \alpha}\|l\|\|\vec{f}\|_{L^{p}\left(\left\{A_{Q}\right\}, \nu\right)}$. By duality,

$$
2^{-\nu \alpha}\left\|l * \varphi_{\nu}\right\|_{L^{p^{\prime}}\left(\left\{A_{Q}^{-1}\right\}, \nu\right)}=2^{-\nu \alpha} \sup _{\vec{f} \in \mathcal{S}_{0}} \frac{\left|\left(l * \varphi_{\nu}\right)(\vec{f})\right|}{\|\vec{f}\|_{L^{p}\left(\left\{A_{Q}\right\}, \nu\right)}} \leq c\|l\|,
$$

i.e., the functional $l * \varphi_{\nu}$ can be associated with a function $\vec{g}_{\nu} \in L^{p^{\prime}}\left(\left\{A_{Q}^{-1}\right\}, \nu\right)$ such that $2^{-\nu \alpha}\left\|\vec{g}_{\nu}\right\|_{L^{p^{\prime}}\left(\left\{A_{Q}^{-1}\right\}, \nu\right)} \leq c\|l\|$. Let $\vec{g}=\sum_{\nu \in \mathbb{Z}} \vec{g}_{\nu} * \theta_{\nu}$, where $\theta$ is as in the atomic decomposition theorem [3, Lemma 5.12] or [2, p. 783], which implies $\widehat{\vec{g}}=\sum_{\nu \in \mathbb{Z}} \widehat{l} \widehat{\varphi}_{\nu} \widehat{\theta}_{\nu}=\widehat{l} \cdot 1$ and so $\vec{g}=l$. Observe that $\vec{g} \in \dot{B}_{p^{\prime}}^{-\alpha \infty}\left(\left\{A_{Q}^{-1}\right\}\right)$ :

$$
\|\vec{g}\|_{\dot{B}_{p^{\prime}}^{-\alpha \infty}\left(\left\{A_{Q}^{-1}\right\}\right)}=\sup _{\nu \in \mathbb{Z}} 2^{-\nu \alpha}\left\|g * \varphi_{\nu}\right\|_{L^{p^{\prime}}\left(\left\{A_{Q}^{-1}\right\}, \nu\right)} \leq c\|l\| .
$$

Thus, the functional $l \in\left[\dot{B}_{p}^{\alpha q}\left(\left\{A_{Q}\right\}\right)\right]^{*}$ can be associated with $\vec{g} \in$ $\dot{B}_{p^{\prime}}^{-\alpha \infty}\left(\left\{A_{Q}^{-1}\right\}\right)$ and $l(\vec{f})=(\vec{f}, \vec{g})$. This completes the proof. 
Summarizing the results of this and the previous section we get the following embeddings of $B$-spaces:

Corollary 6.3. Let $W$ be a matrix weight and $\left\{A_{Q}\right\}_{Q}$ its reducing operators. Let $\alpha \in \mathbb{R}, 0<q<\infty$ and $1 \leq p<\infty$. Then

$$
\begin{aligned}
{\left[\dot{B}_{p}^{\alpha q}(W)\right]^{*} } & \stackrel{(1)}{\subseteq}\left[\dot{B}_{p}^{\alpha q}\left(\left\{A_{Q}\right\}\right)\right]^{*} \stackrel{(2)}{\subseteq} \dot{B}_{p^{\prime}}^{-\alpha q^{\prime}}\left(\left\{A_{Q}^{-1}\right\}\right) \\
& \stackrel{(3)}{\subseteq} \dot{B}_{p^{\prime}}^{-\alpha q^{\prime}}\left(\left\{A_{Q}^{\#}\right\}\right) \stackrel{(4)}{\subseteq} \dot{B}_{p^{\prime}}^{-\alpha q^{\prime}}\left(W^{-p^{\prime} / p}\right),
\end{aligned}
$$

where

- (1) holds if $W$ is doubling of order $p$,

- (2) holds if $W$ is doubling of order $p$,

- (3) holds if $W \in A_{p}, 1<p<\infty$,

- (4) holds if $W^{-p^{\prime} / p}$ is doubling of order $p^{\prime}, 1<p<\infty$.

Also,

$$
\begin{aligned}
{\left[\dot{B}_{p}^{\alpha q}(W)\right]^{*} } & \stackrel{\left(1^{*}\right)}{\supseteq}\left[\dot{B}_{p}^{\alpha q}\left(\left\{A_{Q}\right\}\right)\right]^{*} \stackrel{\left(2^{*}\right)}{\supseteq} \dot{B}_{p^{\prime}}^{-\alpha q^{\prime}}\left(\left\{A_{Q}^{-1}\right\}\right) \\
& \stackrel{\left(3^{*}\right)}{\supseteq} \dot{B}_{p^{\prime}}^{-\alpha q^{\prime}}\left(\left\{A_{Q}^{\#}\right\}\right) \stackrel{\left(4^{*}\right)}{\supseteq} \dot{B}_{p^{\prime}}^{-\alpha q^{\prime}}\left(W^{-p^{\prime} / p}\right),
\end{aligned}
$$

where

- $\left(1^{*}\right)$ holds if $W$ satisfies any of (A1)-(A3),

- $\left(2^{*}\right)$ holds if $W$ is doubling of order $p$,

- $\left(3^{*}\right)$ holds for any matrix weight $W$,

- $\left(4^{*}\right)$ holds if $W^{-p^{\prime} / p}$ satisfies any of (A1)-(A3), $1<p<\infty$.

In terms of a matrix weight $W$ only, (45) and (46) are

$$
\left[\dot{B}_{p}^{\alpha q}(W)\right]^{*} \subseteq \dot{B}_{p^{\prime}}^{-\alpha q^{\prime}}\left(W^{-p^{\prime} / p}\right) \quad \text { if } W \in A_{p}, 1<p<\infty,
$$

and

$$
\left[\dot{B}_{p}^{\alpha q}(W)\right]^{*} \supseteq \dot{B}_{p^{\prime}}^{-\alpha q^{\prime}}\left(W^{-p^{\prime} / p}\right)
$$

if $W, W^{-p^{\prime} / p}$ satisfy any of (A1)-(A3), $1<p<\infty$.

In particular, if $W \in A_{p}$ (and so $W^{-p^{\prime} / p} \in A_{p^{\prime}}$ ), then $\left[\dot{B}_{p}^{\alpha q}(W)\right]^{*} \approx$ $\dot{B}_{p^{\prime}}^{-\alpha q^{\prime}}\left(W^{-p^{\prime} / p}\right)$, otherwise ( $W$ still satisfies any of (A1)-(A3), otherwise there may be a dependence on $\varphi)\left[\dot{B}_{p}^{\alpha q}(W)\right]^{*} \approx \dot{B}_{p^{\prime}}^{-\alpha q^{\prime}}\left(\left\{A_{Q}^{-1}\right\}\right)$, which completes the proof of Theorem A1.

7. Duality of inhomogeneous Besov spaces $b_{p}^{\alpha q}(W)$ and $B_{p}^{\alpha q}(W)$. Recall that the main difference between homogeneous and inhomogeneous spaces is that instead of considering all dyadic cubes, we only consider the ones with side length $l(Q) \leq 1$ and the properties of functions corresponding 
to $l(Q)=1$ are slightly changed. We start with the sequence spaces. Recall the definition of the space $b_{p}^{\alpha q}(W)$.

Definition 7.1 (Inhomogeneous matrix-weighted sequence Besov space $\left.b_{p}^{\alpha q}(W)\right)$. For $\alpha \in \mathbb{R}, 0<q \leq \infty, 1 \leq p<\infty$ and $W$ a matrix weight, the space $b_{p}^{\alpha q}(W)$ consists of all vector-valued sequences $\vec{s}=\left\{\vec{s}_{Q}\right\}_{l(Q) \leq 1}$ such that

$$
\|\vec{s}\|_{b_{p}^{\alpha q}(W)}=\left\|\left\{2^{\nu \alpha}\left\|\sum_{l(Q)=2^{-\nu}}|Q|^{-1 / 2} \vec{s}_{Q} \chi_{Q}\right\|_{L^{p}(W)}\right\}_{\nu \geq 0}\right\|_{l^{q}}<\infty .
$$

Let $\mathcal{R S}^{(I)}$ be the collection of all sequences $\left\{A_{Q}\right\}_{l(Q) \leq 1}$ of positivedefinite operators on $\mathcal{H}$. Similar to the homogeneous case, we introduce the averaging space $b_{p}^{\alpha q}\left(\left\{A_{Q}\right\}\right)$.

DEFInITION 7.2 (Inhomogeneous averaging matrix-weighted sequence Besov space $\left.b_{p}^{\alpha q}\left(\left\{A_{Q}\right\}\right)\right)$. For $\alpha \in \mathbb{R}, 0<q \leq \infty, 1 \leq p \leq \infty$ and $\left\{A_{Q}\right\}_{l(Q) \leq 1}$ $\in \mathcal{R S}^{(I)}$, let

$$
\begin{aligned}
& b_{p}^{\alpha q}\left(\left\{A_{Q}\right\}\right)=\left\{\vec{s}=\left\{\vec{s}_{Q}\right\}_{l(Q) \leq 1}:\right. \\
& \left.\|\vec{s}\|_{b_{p}^{\alpha q}\left(\left\{A_{Q}\right\}\right)}=\left\|\left\{2^{\nu \alpha}\left\|\sum_{l(Q)=2^{-\nu}}|Q|^{-1 / 2} \vec{s}_{Q} \chi_{Q}\right\|_{L^{p}\left(\left\{A_{Q}\right\}, \nu\right)}\right\}_{\nu \geq 0}\right\|_{l^{q}}<\infty\right\} .
\end{aligned}
$$

Let $\vec{s} \in b_{p}^{\alpha q}(W)$. Define $\vec{s}=\left\{\vec{s}_{Q}\right\}_{Q \in \mathcal{D}}$ by setting $\vec{s}_{Q}=\vec{s}_{Q}$ if $l(Q) \leq 1$ and $\vec{s}_{Q}=0$ if $l(Q)>1$. Note that $\vec{s}$ is the restriction of $\vec{s}$ on $b_{p}^{\alpha q}(W)$. Applying (4), we get

$$
\|\vec{s}\|_{b_{p}^{\alpha q}(W)}=\|\vec{s}\|_{\dot{b}_{p}^{\alpha q}(W)} \approx\|\vec{s}\|_{\dot{b}_{p}^{\alpha q}\left(\left\{A_{Q}\right\}\right)}=\|\vec{s}\|_{b_{p}^{\alpha q}\left(\left\{A_{Q}\right\}\right)},
$$

which proves the following proposition.

Proposition 7.3. Let $\alpha \in \mathbb{R}, 1 \leq p<\infty, 0<q \leq \infty$ and let $W$ be $a$ matrix weight with reducing operators $\left\{A_{Q}\right\}_{Q}$. Then

$$
b_{p}^{\alpha q}(W) \approx b_{p}^{\alpha q}\left(\left\{A_{Q}\right\}\right)
$$

in the sense of the norm equivalence.

Note that it is enough to consider reducing operators $A_{Q}$ generated by a matrix weight $W$ only for dyadic cubes of side length $l(Q) \leq 1$, i.e., $\left\{A_{Q}\right\}_{l(Q) \leq 1}$.

Now we establish the duality.

Theorem A3. Let $\alpha \in \mathbb{R}, 1 \leq p<\infty, 0<q<\infty$ and let $W$ be a matrix weight with reducing operators $\left\{A_{Q}\right\}_{l(Q) \leq 1}$. Then

$$
\left[b_{p}^{\alpha q}(W)\right]^{*} \approx b_{p^{\prime}}^{-\alpha q^{\prime}}\left(\left\{A_{Q}^{-1}\right\}\right) .
$$

Moreover, if $W \in A_{p}, 1<p<\infty$, then

$$
\left[b_{p}^{\alpha q}(W)\right]^{*} \approx b_{p^{\prime}}^{-\alpha q^{\prime}}\left(W^{-p^{\prime} / p}\right) .
$$


To prove this theorem one can simply repeat the arguments from Section 3 with proper adjustments (for example, consider sums on $\nu$ taken only over $\nu \geq 0$ ). However, we would like to give a simple proof for the embedding

$$
\left[b_{p}^{\alpha q}\left(\left\{A_{Q}\right\}\right)\right]^{*} \subseteq b_{p^{\prime}}^{-\alpha q^{\prime}}\left(\left\{A_{Q}^{-1}\right\}\right) .
$$

Proof. Let $l \in\left[b_{p}^{\alpha q}\left(\left\{A_{Q}\right\}\right)\right]^{*}$. Let $P$ be the projection from $\dot{b}_{p}^{\alpha q}\left(\left\{A_{Q}\right\}\right)$ to $b_{p}^{\alpha q}\left(\left\{A_{Q}\right\}\right)$ defined by restricting a sequence $\left\{\vec{s}_{Q}\right\}_{Q \in \mathcal{D}}$ to $\left\{\vec{s}_{Q}\right\}_{l(Q) \leq 1}$. Set $\tilde{l}$ by $\widetilde{l}(\vec{s})=l(P \vec{s})$ for each $\vec{s} \in \dot{b}_{p}^{\alpha q}\left(\left\{A_{Q}\right\}\right)$. Then $\widetilde{l} \in\left[\dot{b}_{p}^{\alpha q}\left(\left\{A_{Q}\right\}\right)\right]^{*}$, since

$$
|\widetilde{l}(\vec{s})|=|l(P \vec{s})| \leq\|l\|\|P \vec{s}\|_{b_{p}^{\alpha q}\left(\left\{A_{Q}\right\}\right)} \leq\|l\|\|\vec{s}\|_{\dot{b}_{p}^{\alpha q}\left(\left\{A_{Q}\right\}\right)} .
$$

Then by Lemma 3.3 (or, equivalently, by (17)), $\widetilde{l}$ is represented by $\overrightarrow{\widetilde{t}} \in$ $\dot{b}_{p^{\prime}}^{-\alpha q^{\prime}}\left(\left\{A_{Q}^{-1}\right\}\right)$ such that $\widetilde{l}(\vec{s})=(\vec{s}, \overrightarrow{\widetilde{t}})$ and $\|\overrightarrow{\widetilde{t}}\|_{\dot{b}_{p^{\prime}}^{-\alpha q^{\prime}}\left(\left\{A_{Q}^{-1}\right\}\right)} \leq\|\widetilde{l}\| \leq\|l\|$. Let $\vec{t}=P \overrightarrow{\vec{t}}$. For $\vec{s} \in b_{p}^{\alpha q}\left(\left\{A_{Q}\right\}\right)$ define $\overrightarrow{\vec{s}} \in \dot{b}_{p}^{\alpha q}\left(\left\{A_{Q}\right\}\right)$ as above (thus, $\left.P \overrightarrow{\vec{s}}=\vec{s}\right)$. Then

$$
l(\vec{s})=\widetilde{l}(\overrightarrow{\dot{s}})=(\overrightarrow{\dot{s}}, \overrightarrow{\widetilde{t}})=\sum_{l(Q) \leq 1} \overrightarrow{\dot{s}}_{Q} \overrightarrow{\widetilde{t}}_{Q}+\sum_{l(Q)>1} \overrightarrow{\dot{s}}_{Q} \overrightarrow{\widetilde{t}}_{Q}=\sum_{l(Q) \leq 1} \overrightarrow{\dot{s}}_{Q} \overrightarrow{\widetilde{t}}_{Q}=(\vec{s}, \vec{t}),
$$

since $\overrightarrow{\dot{s}}_{Q}=0$ for $l(Q)>1$. Moreover, $\|\vec{t}\|_{b_{p^{\prime}}^{-\alpha q^{\prime}}\left(\left\{A_{Q}^{-1}\right\}\right)} \leq\|\overrightarrow{\widetilde{t}}\|_{\dot{b}_{p^{\prime}}^{-\alpha q^{\prime}}\left(\left\{A_{Q}^{-1}\right\}\right)} \leq$ $\|l\|$.

Consider a class of functions $\mathcal{A}^{(I)}$ with properties similar to the ones of an admissible kernel: $\Phi \in \mathcal{A}^{(I)}$ if $\Phi \in \mathcal{S}\left(\mathbb{R}^{n}\right)$, supp $\widehat{\Phi} \subseteq\left\{\xi \in \mathbb{R}^{n}:|\xi| \leq 2\right\}$ and $|\widehat{\Phi}(\xi)| \geq c>0$ if $|\xi| \leq 5 / 3$. Recall the inhomogeneous space $B_{p}^{\alpha q}(W)$ from $[5]$.

Definition 7.4 (Inhomogeneous matrix-weighted Besov space $B_{p}^{\alpha q}(W)$ ). For $\alpha \in \mathbb{R}, 1 \leq p<\infty, 0<q \leq \infty, W$ a matrix weight, $\varphi \in \mathcal{A}$ and $\Phi \in \mathcal{A}^{(I)}$, we define the Besov space $B_{p}^{\alpha q}(W)$ to be the collection of all vector-valued distributions $\vec{f}=\left(f_{1}, \ldots, f_{m}\right)^{\mathrm{T}}$ with $f_{i} \in \mathcal{S}^{\prime}\left(\mathbb{R}^{n}\right), 1 \leq i \leq m$, such that

$$
\|\vec{f}\|_{B_{p}^{\alpha q}(W)}=\|\Phi * \vec{f}\|_{L^{p}(W)}+\left\|\left\{2^{\nu \alpha}\left\|\varphi_{\nu} * \vec{f}\right\|_{L^{p}(W)}\right\}_{\nu \geq 1}\right\|_{l^{q}}<\infty .
$$

Analogously, we introduce the averaging space $B_{p}^{\alpha q}\left(\left\{A_{Q}\right\}\right)$.

Definition 7.5 (Averaging matrix-weighted Besov space $B_{p}^{\alpha q}\left(\left\{A_{Q}\right\}\right)$ ). For $\alpha \in \mathbb{R}, 0<q \leq \infty, 1 \leq p \leq \infty, \varphi \in \mathcal{A}, \Phi \in \mathcal{A}^{(I)}$ and $\left\{A_{Q}\right\}_{l(Q) \leq 1} \in$ $\mathcal{R S}^{(I)}$, let

$$
\begin{aligned}
& B_{p}^{\alpha q}\left(\left\{A_{Q}\right\}\right)=\left\{\vec{f}=\left(f_{1}, \ldots, f_{m}\right)^{\mathrm{T}} \text { with } f_{i} \in \mathcal{S}^{\prime}\left(\mathbb{R}^{n}\right), 1 \leq i \leq m:\right. \\
& \left.\|\vec{f}\|_{\dot{B}_{p}^{\alpha q}\left(\left\{A_{Q}\right\}\right)}=\|\Phi * \vec{f}\|_{L^{p}\left(\left\{A_{Q}\right\}, 0\right)}+\left\|\left\{2^{\nu \alpha}\left\|\varphi_{\nu} * \vec{f}\right\|_{L^{p}\left(\left\{A_{Q}\right\}, \nu\right)}\right\}_{\nu \geq 1}\right\|_{l^{q}}<\infty\right\} .
\end{aligned}
$$

Now the remaining results of Sections 4-6 transfer easily to the inhomogeneous Besov spaces by using the properties established in Section 12 
of [5], such as replacing a family $\left\{\varphi_{\nu}\right\}_{\nu \in \mathbb{Z}}$ with $\left\{\varphi_{\nu}\right\}_{\nu \in \mathbb{N}} \cup \Phi$, observing that $\Phi * \vec{f} \in E_{0}$ and summing over $\nu \geq 0$ (or $l(Q) \leq 1$ ) in all sums. In particular, we get

TheOREM 7.6. Let $\alpha \in \mathbb{R}, 0<q \leq \infty, 1 \leq p<\infty$ and let $\left\{A_{Q}\right\}_{l(Q) \leq 1}$ be a doubling sequence (of order $p$ ). Then for $\vec{s}_{Q}(\vec{f})=\left\langle\vec{f}, \varphi_{Q}\right\rangle$,

$$
\|\vec{f}\|_{B_{p}^{\alpha q}\left(\left\{A_{Q}\right\}\right)} \approx\left\|\left\{\vec{s}_{Q}(\vec{f})\right\}_{l(Q) \leq 1}\right\|_{b_{p}^{\alpha q}\left(\left\{A_{Q}\right\}\right)} .
$$

Corollary 7.7. The spaces $B_{p}^{\alpha q}\left(\left\{A_{Q}\right\}\right), B_{p}^{\alpha q}\left(\left\{A_{Q}^{-1}\right\}\right)$ and $B_{p^{\prime}}^{-\alpha q^{\prime}}\left(\left\{A_{Q}^{-1}\right\}\right)$ are independent of the choice of the pair of admissible kernels $(\varphi, \Phi)$ if $\left\{A_{Q}\right\}_{l(Q) \leq 1}$ is doubling (of order $\left.p\right), 1 \leq p<\infty, \alpha \in \mathbb{R}, 0<q \leq \infty$.

LEMmA 7.8. Let $\alpha \in \mathbb{R}, 0<q \leq \infty$ and $1<p<\infty$. If $W$ satisfies any of (A1)-(A3) and $\left\{A_{Q}\right\}_{l(Q) \leq 1}$ is a sequence of reducing operators generated by $W$, then

$$
B_{p}^{\alpha q}(W) \approx B_{p}^{\alpha q}\left(\left\{A_{Q}\right\}\right) .
$$

Theorem A4. Let $\alpha \in \mathbb{R}, 0<q<\infty, 1 \leq p<\infty$ and let $\left\{A_{Q}\right\}_{l(Q) \leq 1}$ be reducing operators of a matrix weight $W$. If $W$ satisfies any of (A1)-(A3), then

$$
\left[B_{p}^{\alpha q}(W)\right]^{*} \approx B_{p^{\prime}}^{-\alpha q^{\prime}}\left(\left\{A_{Q}^{-1}\right\}\right)
$$

If $W \in A_{p}, 1<p<\infty$, then

$$
\left[B_{p}^{\alpha q}(W)\right]^{*} \approx B_{p^{\prime}}^{-\alpha q^{\prime}}\left(W^{-p^{\prime} / p}\right) .
$$

Acknowledgements. The author would like to thank Prof. Michael Frazier for comments and suggestions while examining the paper.

\section{References}

[1] R. Edwards, Functional Analysis: Theory and Applications, Holt, Rinehart and Winston, 1965.

[2] M. Frazier and B. Jawerth, Decomposition of Besov spaces, Indiana Univ. Math. J. 34 (1985), 777-799.

[3] M. Frazier, B. Jawerth and G. Weiss, Littlewood-Paley Theory and the Study of Function Spaces, CBMS Reg. Conf. Ser. Math. 79, Amer. Math. Soc., Providence, RI, 1991.

[4] F. Nazarov and S. Treil, The hunt for a Bellman function: applications to estimates for singular integral operators and to other classical problems of harmonic analysis, Algebra i Analiz 8 (1996), no. 5, 32-162 (in Russian).

[5] S. Roudenko, Matrix-weighted Besov spaces, Trans. Amer. Math. Soc. 355 (2003), 273-314.

[6] - , The theory of function spaces with matrix weights, PhD thesis, Michigan State Univ., 2002.

[7] E. Stein, Harmonic Analysis, Princeton Univ. Press, 1993.

[8] S. Treil and A. Volberg, Wavelets and the angle between past and future, J. Funct. Anal. 143 (1997), 269-308. 
[9] H. Triebel, Theory of Function Spaces, Monogr. Math. 78, Birkhäuser, Basel, 1983.

[10] A. Volberg, Matrix $A_{p}$ weights via S-functions, J. Amer. Math. Soc. 10 (1997), 445-466.

Mathematics Department

Duke University

Durham, NC 27708, U.S.A.

E-mail: svetlana@math.duke.edu

Received July 16, 2002

Final version April 8, 2003 